\title{
Adjusted Bayesian Completion Rates (ABC) Estimation
}

\author{
Ameer Dharamshi ${ }^{1}$ \\ Bilal Barakat ${ }^{2}$ \\ Leontine Alkema ${ }^{3}$ \\ Manos Antoninis ${ }^{2}$ \\ ${ }^{1}$ University of Toronto \\ ${ }^{2}$ Global Education Monitoring Report (GEMR) - UNESCO \\ ${ }^{3}$ University of Massachusetts Amherst
}

September 21, 2021

\begin{abstract}
Estimating school completion is crucial for monitoring SDG 4 on education. The recently introduced SDG indicator 4.1.2, defined as the percentage of children aged 3-5 years above the expected completion age of a given level of education that have completed the respective level, differs from enrolment indicators in that it relies primarily on household surveys. This introduces a number of challenges including gaps between survey waves, conflicting estimates, age misreporting, and delayed completion. Our Adjusted Bayesian Completion Rates (ABC) model addresses these challenges to produce the first complete and consistent time series for SDG indicator 4.1.2, by school level and sex, for 157 countries. The ABC model estimates unobserved true completion rates using a latent $\operatorname{ARIMA}(1,1,0)$ with drift process. The model adjusts observations for late completion and age misreporting effects, and also accounts for survey level differences in bias and non-sampling variance. Validation exercises indicate that the model appears well-calibrated and offers a meaningful improvement over simpler approaches in predictive performance.

Keywords: Bayesian modelling, household surveys, misreporting, school completion, SDG 4
\end{abstract}

\section{Contents}

1 Introduction $\quad 2$

2 Definitions and Data $\quad 4$

2.1 School Completion Rates . . . . . . . . . . . . . . . . . . . . . . . 4

2.2 Retrospective Data . . . . . . . . . . . . . . . . . . . . . . . 4

3 Methods 6

3.1 Model Summary . . . . . . . . . . . . . . . . . . . . . . . . . . 6

3.2 Core Model for the Underlying Trend f . . . . . . . . . . . . . . . . . . . . . 6

3.3 Late Completion . . . . . . . . . . . . . . . . . . . . . . . . . . . . . . 7

3.4 Data Considerations . . . . . . . . . . . . . . . . . . . . . . . . 9

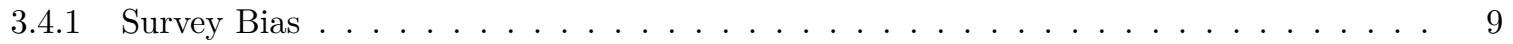

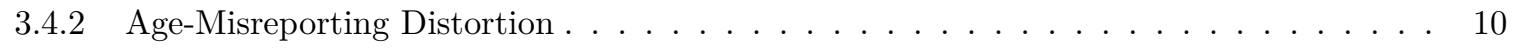

3.4 .3 Variance . . . . . . . . . . . . . . . . . . . . . . . . . 10

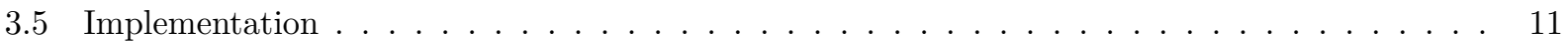

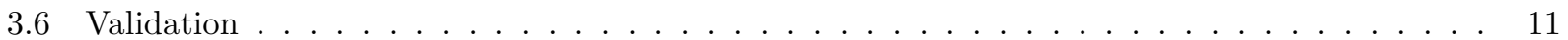

4 Results 11

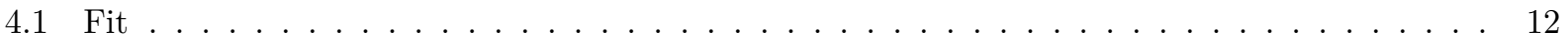

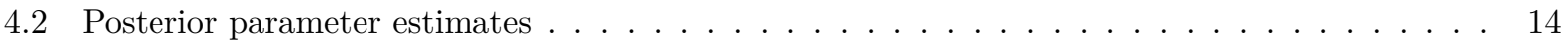


A Model Details $\quad 20$

A.1 Process Model . . . . . . . . . . . . . . . . . . . . . . . . . 20

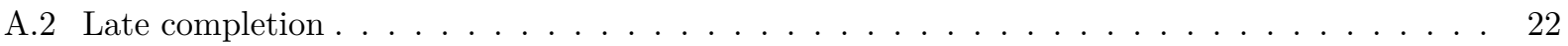

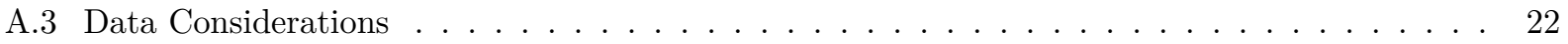

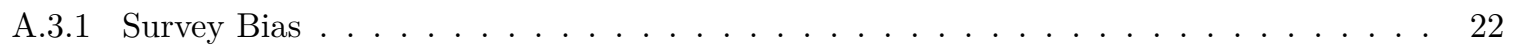

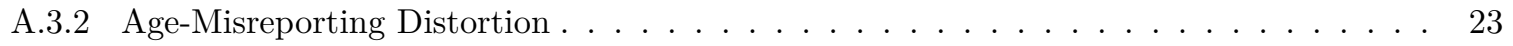

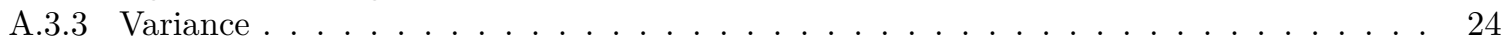

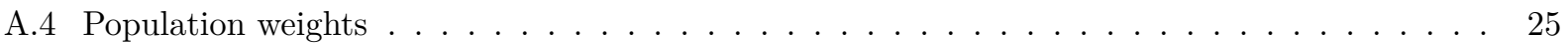

B Implementation Details $\quad 26$

B.1 Data Sources . . . . . . . . . . . . . . . . . . . . . . . . 26

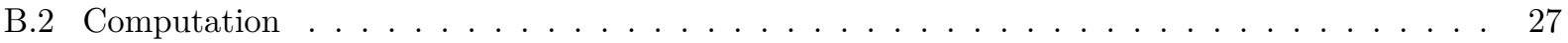

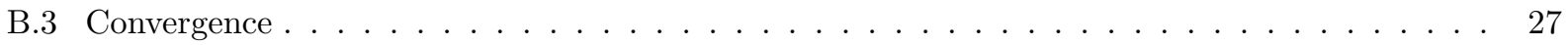

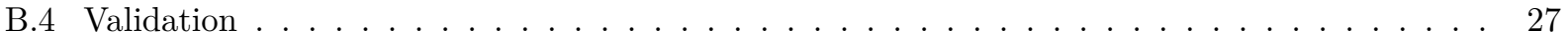

\section{Introduction}

Sustainable Development Goal 4 on education and its monitoring framework have shifted attention away from mere enrolment towards school completion and learning. In keeping with this trend, a new indicator, SDG Indicator 4.1.2 measuring school completion rates at the primary, lower secondary, and upper secondary education levels, has been introduced. Indicator 4.1.2 is officially defined as the "percentage of a cohort of children or young people aged 3-5 years above the intended age for the last grade of each level of education who have completed that grade" (UNESCO Institute of Statistics, 2020). Historically, measurements of school completion have focused on adult populations to capture ultimate adult attainment. Indicator 4.1.2 differs in that it is intended to capture youth school completion with a 3-5 year buffer for minor delays instead of waiting 10-15 years for adult attainment. This will allow for timely readings of progress or stalling in school completion, facilitating faster policy responses.

Traditionally, education monitoring relies on administrative enrolment records provided by individual countries to compute enrolment indicators. Unfortunately, this data often does not track student progression and thus provides limited insight on outcomes. As such, SDG target 4.1 on education completion and target 4.5 on equal access have increased interest in using household survey or census data to monitor education indicators.

In the present circumstance, administrative data on graduates by age or other population characteristics are rarely available, limiting their use in school completion. We thus focus on household surveys and census data, both of which directly ask individuals about total years of schooling and attainment of each level.

Survey data, however, bring their own challenges. Most cross-country comparable household survey programmes conduct a survey in a given country at most every three to five years and the results released at least one year later, generating a considerable time lag. For most countries, multiple surveys are available though they may provide conflicting information. The 2016 Global Education Monitoring (GEM) Report raised the question of reconciling the different sources (UNESCO, 2016). Simply averaging estimates or fitting a standard linear regression trend ignores relevant information. Some sources may show greater variability due to small sample size or other, non-statistical issues that make them less reliable. By itself, this could be accounted for using weighted linear regression. This method still does not recognise, however, that some sources may systematically result in lower or higher estimates relative to others. Such bias can reflect differences in sampling frames or how questions are asked. In addition, some respondents provide information retrospectively and the time that has lapsed increases the risk of errors that need to be corrected. 
Figure 1 illustrates the problem using primary completion rates. It is clear that an assessment of the trend has to consider the relatively larger uncertainty of the 2003 estimate, and that recent Demographic and Health Surveys (DHS) and Multiple Indicator Cluster Surveys (MICS) surveys systematically differ in their baseline. Comparing them directly, or always adopting the 'latest available' as the best estimate would lead to the conclusion that there have been large jumps in completion in a short amount of time, in opposite directions.

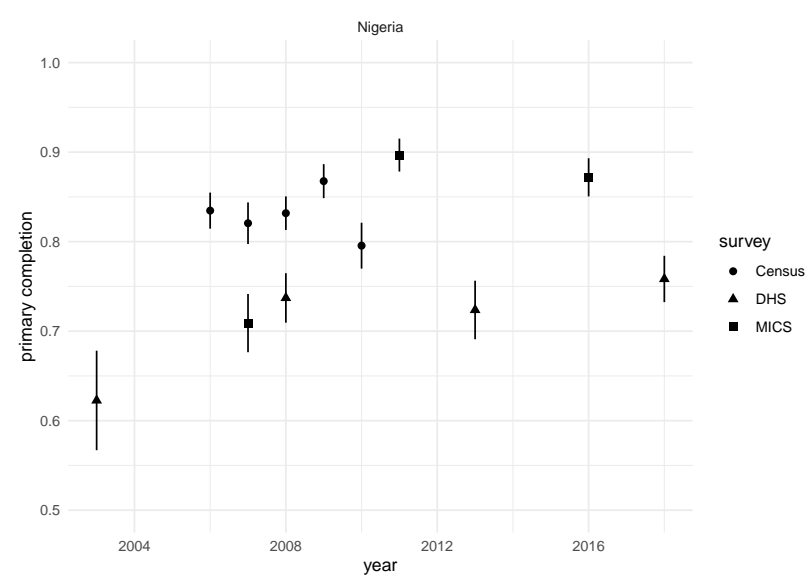

Figure 1: Primary school completion rate in Nigeria five years above nominal age for final primary grade, from different surveys, with estimated $95 \%$ uncertainty intervals. N.B. y-axis starts at 0.5.

These challenges also constrain the calculation of consistent trends in completion rates for regional aggregates because the set of countries with observations in a given year changes over time.

The international health community faced a similar challenge in measuring indicators, such as under 5 mortality or maternal mortality rates, based on multiple sources. The UN Inter-agency Group for Child Mortality Estimation adopted a consensus model to generate annual estimates for under 5 (Alkema and New, 2014) and neo-natal mortality (Alexander and Alkema, 2018) in each member state. The Inter-Agency Group for Maternal Mortality Rates followed a similar process (Alkema et al., 2016).

Here, we introduce the Adjusted Bayesian Completion Rates (ABC) model that builds on these models for health indicators, but is fully adapted to estimating school completion rates. As a result, it is simpler in some respects, but more complex in others. The ABC model seeks to address concerns about limited data by reconstructing historical completion rates from the available surveys and recognizing the increased error associated with retrospective series. We also explicitly address late completion, and age-misreporting concerns stemming from limited survey respondent numeracy skills in some regions. Such adjustments permit the ABC model to consolidate survey data into a smooth underlying trend in completion rates from which the estimated true annual completion rates for each country can be extracted.

Our ABC model is the first to estimate school completion rates as defined by SDG Indicator 4.1.2 as opposed to the less timely educational attainment among adults. In addition to the completion rate for the age group underlying the official Completion Rate indicator, we also estimate the 'ultimate' rate of school completion. By addressing the various data quality concerns associated with survey data, our completion estimates are also less sensitive to individual surveys, in which years they are conducted, and which survey happens to be the latest available for a given country.

We begin this paper by formally establishing the official definition of the Completion Rate indicator and the associated notation before presenting the structure of the model and how it addresses the specific challenges posed by estimating this indicator. We then present a first set of estimates along with an assessment of their quality and performance against more simplistic approaches. Finally, we conclude with a summary of the outcomes and challenges associated with the ABC model.

The analyses in this paper are based on a consolidated collection of 616 microlevel datasets on school 
completion from 157 countries. The sources are described in detail in Appendix B.1.

\section{Definitions and Data}

\subsection{School Completion Rates}

The agreed SDG indicator of school completion measures completion among individuals who are between three and five years above the theoretical age for the final grade of the education level in question (UNESCO Institute of Statistics, 2020). This theoretical final grade age is the age of a child who starts school at the official school entry age and progresses one grade each year. We refer to this age as $a_{0}$. Then, we refer to age $a_{0}+3$ as $a_{3}, a_{0}+5$ as $a_{5}$, and generally to $a_{0}+n$ as $a_{n}$. Note that the age value corresponding to $a_{0}$ is defined by a given school system and thus varies across countries and in a few countries, over time. For simplicity, however, we omit subscripts for country and year and understand that the numeric value of $a_{0}$ depends on the country-year in question.

Ideally, the most timely observation of completion would be based on individuals one year above $a_{0}$. The reason a three age interval is considered instead is to smooth out variation resulting from the potentially small sample size of any given birth cohort in household survey data. The age bracket is shifted up by two years to offer a 'grace period' for delayed completion. Timely entry and progression without repetition are important goals in their own right. Nevertheless, even though children who start school late and/or repeat grades often suffer an elevated risk of drop-out, many of them do eventually complete school. Accordingly, the completion rate indicator, by focusing on the age group 3 to 5 years above the final grade, seeks to abstract away from the question of timeliness to some extent and capture all completion that is not unreasonably delayed.

We now define $C_{a, c, y}$ as the observed average completion at age $a$ in a given country $c$ in year $y$, such as 15-year-olds in Nigeria in 2010. As each combination of primary, lower secondary, and upper secondary school levels and female, male, and total populations are modeled independently, the level and sex indices have been omitted for simplicity. The 'Completion Rate' indicator, $C R_{c, y}$ is defined as a population-weighted average completion rate for individuals in the interval $\left[a_{3}, a_{5}\right]$. However, we find that the variation in population within the age bracket does not provide useful information regarding school completion, but rather, adds an extra layer of complexity for any near-term projections given that population projections are typically based on five-year aggregates. Instead, we propose to adjust the 'Completion Rate' indicator to an unweighted average. While we recognize that any definitional adjustment may cause marginal departures from observed completion rates, for the purposes of estimation and understanding trends, we use the following adjusted 'Completion Rate' indicator:

$$
C R_{c, y}^{*}=\frac{1}{3} \sum_{i=3}^{5} C_{a_{i}, c, y}
$$

We provide further justification for the definitional adjustment in Appendix A.4.

\subsection{Retrospective Data}

Observations of school completion rates are collected from censuses and household survey programs by aggregating individual level responses to questions on either an individual's total number of years of school completed or more directly what levels have been completed. As these nationally representative household surveys are conducted relatively infrequently, it is necessary to exploit as much information as possible from each round. If each survey only contributed estimates for the survey year for those individuals observed during the nominal age range for the Completion Rate indicator, many countries would have too few observations to perform any kind of robust statistical trend estimation. In the most extreme cases, there is only one survey for a country and thus one observation of individuals in the indicator age bracket. 
However, censuses and household survey programs do not just ask individuals in the $\left[a_{3}, a_{5}\right]$ age bracket about their education status, rather individuals of all ages are asked about their levels and years of school completion. Thus, one solution to the infrequent survey challenge is to take into account the rate of school completion reported by older cohorts who were outside of the indicator age bracket at the time of survey.

In particular, suppose for simplicity that all those who do complete school do so by the time they reach the top of the age bracket. If the age bracket for the completion rate is 14 to 16 in a given country, then a survey in the year 2015 allows for the calculation of the 2015 completion rate based on the 14- to 16-year-olds in the sample. In addition, completion among 17- to 19-year-olds in the sample may be taken as a proxy for the completion rate among 14- to 16-year-olds three years prior, in 2012. In this way, a single survey contributes completion rate estimates for a series of years, as illustrated in Figure 2. Note that the overlapping series of retrospective completion rates make it unambiguously clear that differences between surveys are often not driven by true changes in the years between the surveys, but reflect different baseline bias. That is, some surveys give systematically higher or lower estimates of completion than others.

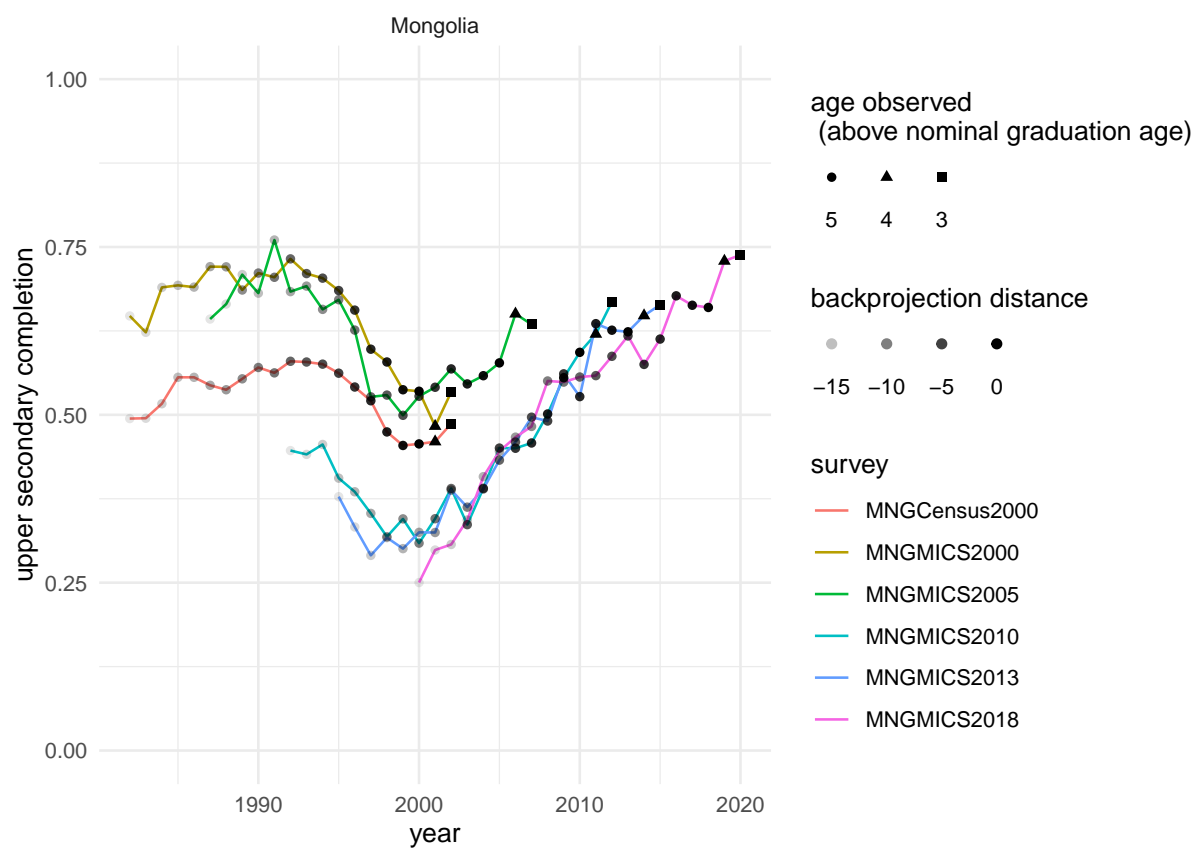

Figure 2: Retrospective series of completion from different surveys. Observations are aligned with the time axis according to the year in which they are at age five years above the nominal age for the final grade. Triangles and squares indicate observations made at younger ages. Faintness indicates retrospective observations of older cohorts.

In general, $C_{a+x, c, y} \approx C_{a, c, y-x}$. However, one way in which this correspondence fails is if there are many individuals completing school between the ages of $a$ and $a+x$. Intuitively, primary school completion among 15-year-olds in 2010 is not informative of primary school completion among 5-year-olds in 2000, for example.

If we were to assume that all completion occurs by some threshold age $a^{\prime}$, the correspondence between $C_{a+x, c, y}$ and $C_{a, c, y-x}$ for $a \geq a^{\prime}$ is more robust but still may not be perfect in general, i.e. observed completion of a given cohort may change at different ages. In particular, completers and non-completers may have systematically different mortality and migration rates. To limit these effects, we reconstruct completion rates only for individuals in a 20 year range starting at the bottom of the nominal age bracket (i.e. from $a_{3}$ up to $\left.a_{23}\right)$ and anchor the correspondence to $a_{5}$. 


\section{Methods}

The objective of the $\mathrm{ABC}$ model is to consolidate observations from different surveys, provide estimates for years without a survey, and allow for short-term 'now-casts' of current completion rates.

Before specifying the model, we recognize that across different levels of schooling and countries, observed completion rates cover practically the entire possible range from $0 \%$ to $100 \%$. To account for outcomes constrained to, but spread across the entire [0,1] interval, we model observations $K_{a, c, y}=\Phi^{-1}\left(C_{a, c, y}\right)$, where $\Phi^{-1}$ is the probit function.

\subsection{Model Summary}

Without loss of generality, we parametrise the model in terms of the top of the age interval for the Completion Rate indicator, that is, we take $a_{5}$ as the reference age. Let $\kappa_{c, y}=\Phi^{-1}\left(\Gamma_{c, y}\right)$ refer to the unknown true completion rate for the $a_{5}$ cohort in country $c$ and year $y$ at the outcome scale $\left(\Gamma_{c, y}\right)$ and transformed scale $\left(\kappa_{c, y}\right)$ respectively.

We then assume that $K_{i}$, the observed probit completion rate relating to age $a[i]$, country $c[i]$, year $y[i]$, and originating from survey $s[i]$ is distributed as follows:

$$
K_{i} \mid \kappa_{c[i], y[i]}, \beta_{s[i]}, \tau_{c[i]}, \phi_{a[i], c[i]}, \nu_{i}, \omega_{a[i], s[i]} \sim \mathcal{N}\left(\kappa_{c[i], y[i]}+\beta_{s[i]}-\tau_{c[i]} \cdot \mathbb{1}_{5 \mid a[i]}+\phi_{a[i], c[i]}, \quad \nu_{i}^{2}+\omega_{a[i], s[i]}^{2}\right)
$$

where $\kappa_{c[i], y[i]}$ is the 'true' probit completion rate for the $a_{5}$ cohort in the respective country and year, $\beta_{s[i]}$ refers to the survey bias, $\tau_{c[i]}$ is a distortion due to age-misreporting occurring when $a[i]$ is a multiple of 5 , $\phi_{a[i], c[i]}$ is the late (relative to $a_{5}$ ) completion adjustment, and finally, $\nu_{i}^{2}$ and $\omega_{a[i], s[i]}^{2}$ refer to the sampling and non-sampling variances respectively.

The model is structured in two stages. The first is the process model governing the changes in underlying true probit completion rates over time. We discuss the process model in detail in Section 3.2. The second describes through Equation (2) how the underlying true $a_{5}$ probit rates relate to the observed data. This relationship is comprised of tangible delays in completion, and various data effects including survey bias and age-misreporting. Late completion is described in Section 3.3 followed by a discussion of the data effects in Section 3.4.

After extracting the true completion rates, $\kappa_{c[i], y[i]}$, from the model, we can estimate the true completion rate indicator as:

$$
\widehat{C R^{*}}{ }_{c, y}=\frac{1}{3} \sum_{a=a_{3}}^{a_{5}} \Gamma_{c, y}=\frac{1}{3} \sum_{a=a_{3}}^{a_{5}} \Phi\left(\kappa_{c, y}+\phi_{a, c}\right) .
$$

Also of interest is the 'ultimate cohort completion', which in our specification is assumed to be reached within 8 years after the nominal age for the final grade and therefore is proxied by $\Phi\left(\kappa_{c, y}+\phi_{a_{8}, c}\right)$.

\subsection{Core Model for the Underlying Trend}

Based on an understanding of the underlying social and policy processes determining completion rates, we wish to allow for the possibility that outcomes in a given year can have both short- and long-term repercussions. A specification in terms of first differences, that is, in changes in completion, better captures our intuition regarding the long-term persistence of shocks. In particular, it is reasonable as a baseline assumption that after a 'lost decade' of exceptionally poor outcomes, the average growth in completion will eventually return to its long-run trend. However, while it is certainly possible to make up for lost time, there is no compelling 
reason to think that the expected level of completion will eventually return to where it would have been in the absence of the crisis period.

Our core model for $\kappa_{c, y}$ is an $\operatorname{ARIMA}(1,1,0)$ with drift process. In addition to meeting the above requirements, the $\operatorname{ARIMA}(1,1,0)$ specification captures a year-over-year autocorrelation relationship, reflecting the possibility of multi-year educational development enablers or hurdles outside of the long-term drift:

$$
\begin{aligned}
\Delta \kappa_{c, y}=\kappa_{c, y}-\kappa_{c, y-1} & =\gamma_{c}+\rho_{c} \Delta \kappa_{c, y-1}+\epsilon_{c, y}, \\
\epsilon_{c, y} \mid \sigma_{\epsilon} & \sim \mathcal{N}\left(0, \sigma_{\epsilon}^{2}\right),
\end{aligned}
$$

with priors:

$$
\begin{aligned}
\sigma_{\epsilon} & \sim \operatorname{Gamma}(2,0.1), \\
\rho_{c} \mid \tau, \lambda_{c} & \sim \mathcal{N}^{+}\left(0, \tau^{2} \lambda_{c}^{2}\right), \\
\tau \mid \tau_{0} & \sim \mathcal{N}^{+}\left(0, \tau_{0}^{2}\right), \\
\lambda_{c} & \sim t_{4}^{+}(0,1) \\
\gamma_{c} \mid \alpha_{\gamma}, \beta_{\gamma} & \sim \log -\operatorname{normal}\left(\alpha_{\gamma}, \beta_{\gamma}\right), \\
\alpha_{\gamma} & \sim \mathcal{N}(0,1), \\
\beta_{\gamma} & \sim \mathcal{N}^{+}(0,1) .
\end{aligned}
$$

The long-term drift $\gamma_{c}$, is expected to be positive, implying an eventual convergence to $100 \%$ completion, including for upper secondary. Educational expansion is the SDG target, and is both a logical requirement over the long term and the general rule empirically. The log-normal distribution is thus selected to reflect the positive constraint while preferring conservative drift estimates, but still allowing for the possibility of faster growth outcomes if there is strong evidence. The parameters of the log-normal distribution are assigned vague normal priors.

Some countries however, have experienced departures from consistent growth as seen with Mongolia in Figure 2. This behaviour of short- to medium-term shocks sustained over a number of years is captured through the country-specific autoregressive coefficient, $\rho_{c}$. We note that the autoregressive structure of an ARIMA $(1,1,0)$ specification could model consistent growth through cascading shocks, creating a possible redundancy with the long-term drift, $\gamma_{c}$. Given that there is substantive interest in understanding the differences in strength of consistent growth between countries, the specification should prefer to model consistent growth with $\gamma_{c}$ instead of with $\rho_{c}$. To reflect that $r h o_{c}$ is only to be relevant in the presence of medium-term shocks that are only experienced by a subset of countries, we assign $\rho_{c}$ a horseshoe prior to shrink unneeded $\rho_{c}$ terms towards zero (Carvalho et al., 2009). Assuming a prior assumption that $30 \%$ of countries will have a relevant $\rho_{c}$ term and a $t_{4}^{+}(0,1)$ distribution on the country-specific horseshoe parameter, $\lambda_{c}$, we set the hyperparameter $\tau_{0}=0.01$ following the procedure discussed in Piironen and Vehtari (2017). Appendix A.1 provides additional comments on both the horesehoe specification and the process model as a whole.

Given that the model is structured in two stages, we employ a vague boundary avoiding prior (Chung et al., 2013, Stan Development Team (2020b)) for $\sigma_{\epsilon}$ to discourage the scenario where the underlying $\kappa$ process exhibits little to no variability. Since the true completion rates are unobserved, a value of $\sigma_{\epsilon}$ approaching zero could be consistent with the likelihood if the variance of the data generating model in Equation (2) expands to entirely compensate for the variability in observations.

\subsection{Late Completion}

In a number of countries where delays in school entry and progression are severe, even the 'grace period' allowed by the shifted age bracket is not sufficient to ensure that $C_{a_{3}, c, y}$ already represents ultimate completion 


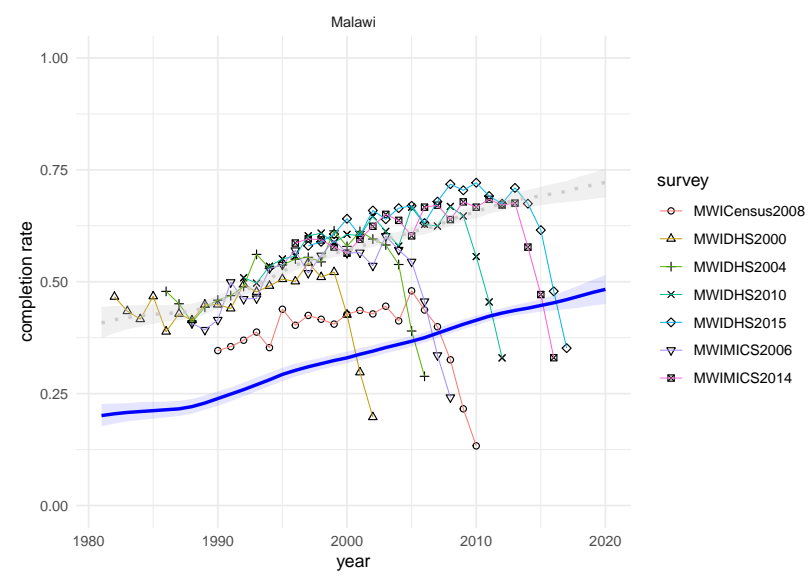

Figure 3: Observed and backcast values of age-specific primary completion in Malawi. The fitted Completion Rate indicator and ultimate completion are indicated by the blue and grey lines respectively.

of the cohort in question and equals $C_{a_{5}, c, y+2}$. In other words, some individuals complete school during the age interval $\left[a_{3}, a_{5}\right]$ and, in some cases, even beyond. This is clearly evident in the example in Figure 3. Observations at ages $a_{3}$ and $a_{4}$ consistently display lower completion than observations at age $a_{5}$.

For a single cross-sectional age profile of completion from one survey, such a pattern would not establish late completion but could, in principle, also arise from a decline in completion between successive cohorts. However, overlaying the retrospective completion rates from several surveys, as in Figure 3, amounts to an implicit pseudo-cohort analysis that shows that ultimate completion suffered no such decline. Instead, the completion observed in a given survey for some pseudo-cohort depends on the age at which it is observed, even at ages above $a_{3}$. Indeed, in this case it is evident that late completion continues even past $a_{5}$.

It is necessary, therefore, to model the age profile of observed completion, in a way that allows for late completion in addition to the error associated with retrospective observations. As a parsimonious but flexible specification, we model the late completion effect $\phi_{a, c}$ as a piece-wise linear function in the probit space with two segments as follows:

$$
\phi_{a, c}= \begin{cases}\left(a-a_{5}\right) \cdot \lambda_{1 c} \cdot \mathbb{1}_{l \mid c} & \text { if } a \in\left\{a_{3}, a_{4}\right\} \\ \min \left(3, a-a_{5}\right) \cdot \lambda_{2 c} \cdot \mathbb{1}_{v l \mid c} & \text { if } a \geq a_{5}\end{cases}
$$

The first case specifies completion within the $\left[a_{3}, a_{5}\right]$ interval potentially being lower by a country-specific value $\lambda_{1 c}$ per year. The second case models additional very late completion beyond the CR indicator interval with a country-specific slope, $\lambda_{2 c}$, accumulating over three years to the age of 'ultimate cohort completion', $a_{8}$. Visually, the age specification is described by Figure 4 .

Both late completion effects are subject to indicator variables dictating the presence or absence of the effect for the given country. Briefly, highly developed education systems have limited or negligible delayed completion beyond the three year grace period offered by the indicator age bracket. To reflect this fact, those countries with median observed completion rates above 0.95 are assumed to not have structural late completion and instead any minor dips are considered noise. However, if for a country with close-to-universal completion, the observations for $\left[a_{3}, a_{5}\right]$ are consistently below those of $\left[a_{5}, a_{7}\right]$ across surveys, shorter-term late completion (ie. $\lambda_{1} c$ ), is estimated. Additional details of the procedure for identifying the presence of late completion is provided in Appendix A.2. The country specific parameters, $\lambda_{1 c}$ and $\lambda_{2 c}$ are modelled hierarchically with the following priors: 


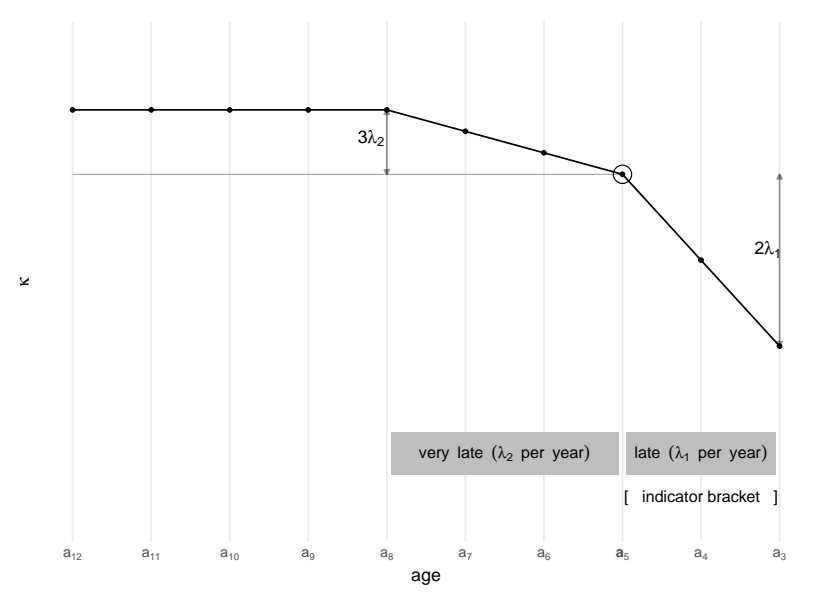

Figure 4: Piece-wise linear model of age-profile of completion. Note that age is decreasing on the x-axis to match a retrospective data series.

$$
\begin{aligned}
\lambda_{1 c} \mid \sigma_{\lambda_{1}} & \sim \mathcal{N}^{+}\left(0, \sigma_{\lambda_{1}}^{2}\right) \\
\lambda_{2 c} \mid \sigma_{\lambda_{2}} & \sim \mathcal{N}^{+}\left(0, \sigma_{\lambda_{2}}^{2}\right) \\
\sigma_{\lambda_{1}} & \sim \mathcal{N}^{+}(0,1) \\
\sigma_{\lambda_{2}} & \sim \mathcal{N}^{+}(0,1)
\end{aligned}
$$

\subsection{Data Considerations}

In addition to the real year-over-year changes in completion and delays in completion, there are consequences of using household surveys that must be accounted for. The three components of these data considerations are survey bias, age-misreporting, and differences in variability.

\subsubsection{Survey Bias}

Given that there currently is not a 'gold standard' for school completion data and that in many countries, even the census is not robust, absolute survey bias cannot be modelled without introducing strong assumptions. However, modelling relative bias allows the model to understand systematic differences between surveys even in periods where retrospective series constructed from different surveys do not overlap. This relative structure induces a sum-to-zero constraint on each country's collection of survey bias terms. Thus, to model $\beta_{c}$, the vector of country c's $S$ survey bias terms, $\beta_{s}$, we parameterize in terms of $\beta_{c}^{*}$, a vector of $S-1$ elements and assume the following:

$$
\begin{aligned}
\beta_{c} & =A_{S} \cdot \beta_{c}^{*} \\
\beta_{c}^{*} \mid \sigma_{\text {bias }} & \sim \operatorname{Cauchy}\left(0, \sigma_{\text {bias }}\right) \\
\sigma_{\text {bias }} & \sim \mathcal{N}^{+}\left(0,0.25^{2}\right)
\end{aligned}
$$

where $A_{S}$ is an $S \times(S-1)$ matrix with elements $a_{i j}$ constrained such that $\forall j \in 1, \ldots, S-1, \sum_{i=1}^{S} a_{i j}=0$, and $\forall i \in 1, \ldots, S, \sum_{j=1}^{S-1}\left|a_{i j}\right|=1$. The first constraint enforces the sum-to-zero constraint using the columns of $A_{S}$, and the second constraint propagates the symmetric Cauchy $\left(0, \sigma_{\text {bias }}\right)$ prior from $\beta_{c}^{*}$ to $\beta_{c}$. The Cauchy distribution is selected here to address the possibility of potentially extreme bias in specific surveys. Additional details, including the specific $A_{S}$ selected, are provided in Appendix A.3.1. 


\subsubsection{Age-Misreporting Distortion}

It is well known that in developing country settings, respondents' ages may be misreported, leading to an overrepresentation of ages that are multiples of five. It is also known that this behaviour correlates with low numeracy skills. Accordingly, it is plausible that cohorts with ages that are multiples of five at the time of a survey may have understated school completion rates due to the overrepresentation of individuals with low numeracy skills that have misreported their ages. When reconstructing retrospective data, this would manifest as anomalous drops in completion every 5 years.

Indeed, we see clear evidence of this in Figure 5, for example. Here, as in a number of other cases, reported primary school completion is lower among those whose reported age is a multiple of five. This is what would be observed if those who did not complete primary school are more likely to round their age.

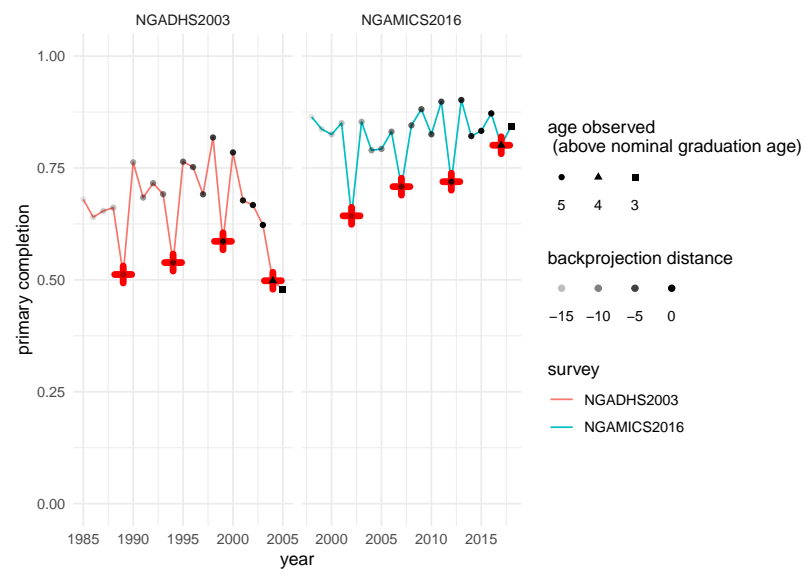

Figure 5: Observed and backcast values of age-specific primary completion in Nigeria. Plus signs indicate observations based on respondents reporting their age at time of survey as a multiple of 5 .

Retrospective observations that represent a reported 'round' age group at the time of survey are coded with an indicator variable. Observations where this indicator equal 1 are subject to an additional term $\tau_{c}$ in Equation (2) that accounts for the potential distortion in country $c$ due to age-misreporting. This distortion is parsimoniously modeled as being rare, but potentially large with the following hierarchical structure:

$$
\begin{aligned}
\tau_{c} \mid \lambda_{\tau} & \sim \operatorname{Exp}\left(\lambda_{\tau}\right) \\
\lambda_{\tau} & \sim \mathcal{N}^{+}\left(0,50^{2}\right)
\end{aligned}
$$

Additional discussion is in Appendix A.3.2.

\subsubsection{Variance}

To account for differences in observation specific sampling variation between surveys (and between age groups), sampling variances, $\nu_{i}$, are estimated a priori and provided as input. As survey reports do not provide sampling errors for school completion rates, $\nu_{i}$ is estimated from the micro-data, applying the clustered Jackknife procedure used to generate the published DHS standard error estimates for other indicators (The Demographic and Health Surveys Program, 2012).

We also assume that the observation for age $a$ from a specific survey $s$ is subject to an independent nonsampling error, $\omega_{a[i], s[i]}$. We noted previously that retrospective data may face increasing uncertainty as time passes due to different mortality and migration rates between completers and non-completers. By restricting the age range for reconstruction to $\left[a_{3}, a_{23}\right]$, the effect of mortality is mostly limited outside of 
jurisdictions with high young adult mortality rates stemming from high HIV/AIDS prevalence. In general, differential migration between completers and non-completers is expected to have a larger impact within the age range. To reflect the increasing uncertainty as the retrospective distance increases, we first estimate an underlying survey level value $\omega_{s}$ and then scale it linearly with the retrospective estimation distance to reflect the increased uncertainty as time passes. Specifically, $\omega_{a, s}^{2}=\left(1+0.05 \cdot \max \left(0, a-a_{5}\right)\right) \cdot \omega_{s}^{2}$. In principle, if not only the migration intensity, but also the migration age schedule differs between completers and non-completers, the magnitude of the retrospective estimation error could be a nonlinear function of the elapsed time $x$ or equivalently, of age at the time of survey. In practice, in the absence of a priori information on this effect, we assume that the uncertainty associated with retrospective estimation increases linearly with age. Further details are presented in Appendix A.3.3.

\subsection{Implementation}

The model parameters are estimated in a Bayesian framework using R. Samples from the posterior distribution are generated with the assistance of the Stan package (Carpenter et al., 2017, Stan Development Team $(2020 \mathrm{c})$ ). Four chains are run in parallel for each level and sex combination. Each chain consists of 3,000 burn-in iterations, and 3,000 samples. Due to memory constraints, the final sample is thinned to 1,000 per level and sex combination. We check convergence using the standard diagnostic checks including trace plots, pairs plots, and the Gelman and Rubin diagnostic (Gelman and Rubin, 1992, Vehtari and Gelman et al. (2020), Stan Development Team (2020a)). See Appendix B for additional implementation details.

\subsection{Validation}

To assess the performance of the model, we consider two different out-of-sample validation exercises. First, we conduct a 'leave one survey out' validation. Specifically, all observations based on the latest survey (including backcast values) for each country with more than one survey are omitted from the estimation of the models, and predicted values for these values are obtained. Such a validation is designed to mimic an intended use case of the model, that is, comparing the output to a new survey. As entire surveys are left out, no survey bias is estimated for the test surveys and so we compare the left out values to the appropriate $\kappa_{c, y}$, adjusted for age-misreporting and late completion, by computing mean squared errors (MSE) and mean absolute errors (MAE).

The second exercise leaves out two random observations from each survey, provided each survey has at least 5 observations. Notably, this does not completely remove any surveys from the data and so bias terms for all surveys can be computed. For this test, we also compute the MSE and MAE between the left out observations and the respective $\kappa_{c, y}$ adjusted for age-misreporting, late completion, and survey bias. We also compute model bias, and coverage of prediction intervals defined as $n^{-1} \sum_{i=1}^{n} \mathbb{1}_{l_{i} \leq y_{i} \leq u_{i}}$ where $n$ is the number of observations in the test set, $i$ is the current observation, and $l_{i}$ and $u_{i}$ are the lower and upper bounds respectively of the prediction interval.

\section{Results}

The model output is illustrated by the country-level results shown in Figure 6 for a selection of countries at three different levels of schooling. The presented examples have been selected to illustrate a variety of scenarios. The results appear sensible, capturing late completion where appropriate, and with projected uncertainty greater when fewer or even only a single survey was available, for instance. Particular attention should be given to the Nigeria, Rwanda, Bhutan, and Armenia examples.

As the country with the single most surveys, the observations for Nigeria appear incredibly noisy. However, as observed in Figure 5, much of this 'noise' is actually the manifestation of age-misreporting. The ABC model considers this information and produces estimates with greater certainty than the underlying data might suggest at face value. Rwanda presents an example of the model's ability to adapt to non-standard 
patterns. While the long-term trend is positive, the effects of the 1994 genocide are clearly visible as a large drop in completion observed and captured by the model. Bhutan presents a single survey case. Given the single survey presents remarkable improvements in completion, a strong positive trend is produced. This is offset however, by significant uncertainty throughout the series. Finally, Armenia demonstrates the behaviour when one survey greatly deviates from the rest, whether as a result of design or implementation problems, or inconsistent coding during analysis. This divergent survey does contribute information to the estimated true completion rates, but does not dramatically pull down the estimates. The Cauchy distribution for survey bias permits large survey biases in rare instances such as this that would be irreconcilable if an alternative such as a Normal distribution had been selected.

\section{$4.1 \quad$ Fit}

In the present case, it is not clear what benchmark the ABC model should be compared to by default as there is no previous attempt at modelling the same outcomes using a simpler specification. We thus consider a 'simple' statistical model in which the $\kappa$ for a given country is a linear function of an intercept and slope over time. In other word, it fits a plain probit curve to the $C_{i}$, without taking into account differences in sampling variation, survey bias, common parameter distributions or any of the advanced data considerations specified in the ABC model.

Results from the 'leave one survey out' validation exercise are presented in Table 1. The mean squared errors (MSE) and mean absolute error (MAE) of the ABC and simple model are both presented multiplied by 100 for ease of reading. The ABC model offers a meaningful and worthwhile improvement on the simple specification. This is despite the fact that the bias of the target surveys are not exploited, since empirically, the survey family is not informative of the bias to be expected of a given individual survey (see Figure 9). On the whole, the advantage of the ABC model is higher for lower levels of schooling where effects such as late completion appear more pronounced. Similarly, the model tends to perform better on female and total population data.

Table 1: ABC 'leave one survey out' validation results.

\begin{tabular}{llrrrr}
\hline Level & Sex & $\begin{array}{r}\text { MSE } \times \text { 100 } \\
(\text { ABC) }\end{array}$ & $\begin{array}{r}\text { MSE } \times \text { 100 } \\
(\text { simple })\end{array}$ & $\begin{array}{r}\text { MAE } \times \text { 100 } \\
(\text { ABC) }\end{array}$ & $\begin{array}{r}\text { MAE } \times \text { 100 } \\
(\text { simple })\end{array}$ \\
\hline prim & female & 0.51 & 0.82 & 3.87 & 5.07 \\
prim & male & 0.47 & 1.05 & 3.99 & 5.97 \\
prim & total & 0.46 & 0.88 & 3.72 & 5.32 \\
lsec & female & 0.50 & 0.67 & 4.62 & 5.39 \\
lsec & male & 0.67 & 1.01 & 5.53 & 6.83 \\
lsec & total & 0.53 & 0.77 & 4.82 & 5.89 \\
usec & female & 0.79 & 0.92 & 6.14 & 6.65 \\
usec & male & 1.05 & 1.27 & 7.45 & 8.33 \\
usec & total & 0.84 & 1.00 & 6.40 & 7.11 \\
\hline
\end{tabular}

The results from the random out-of-sample exercise are presented in Table 2. The bias values suggest the model does not experience significant bias. The coverage values reported are in general close to the nominal level though there are slightly fewer out-of-sample observations outside of the prediction intervals than expected. We note that the tendency to produce conservative predictions is consistent with the in-sample findings in Appendix B.4 and is preferred over the contrary. 


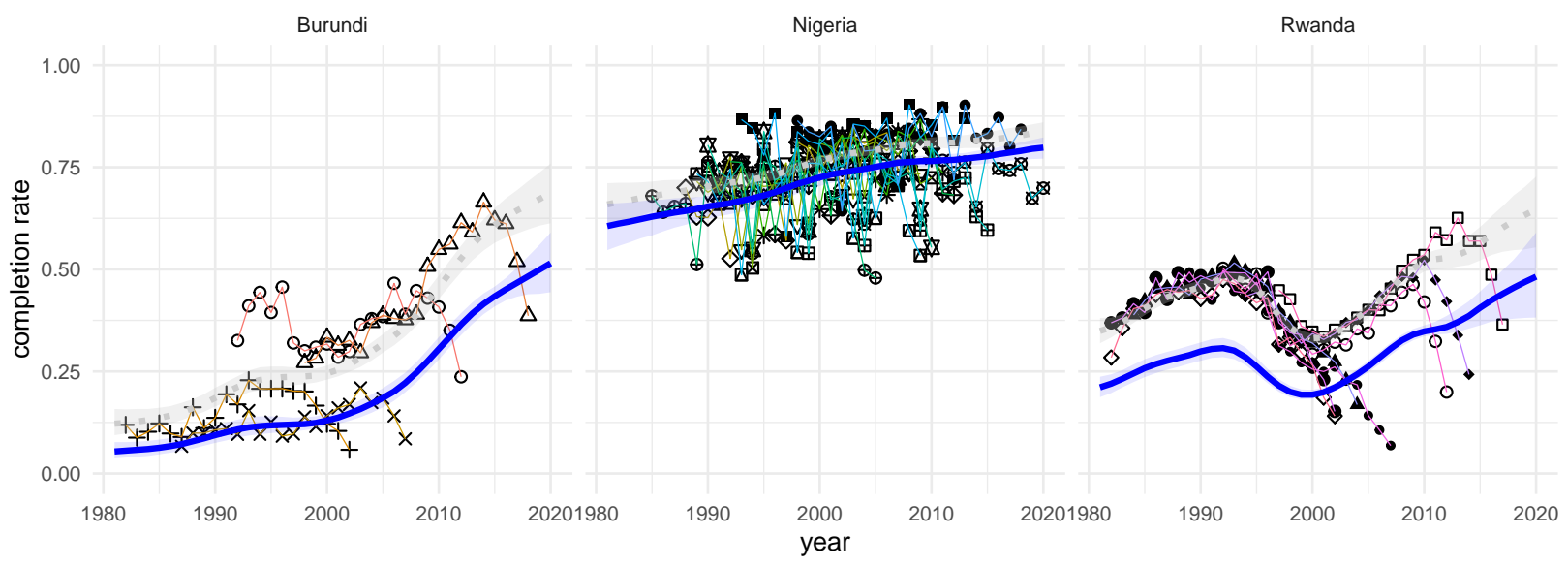

(a) Primary

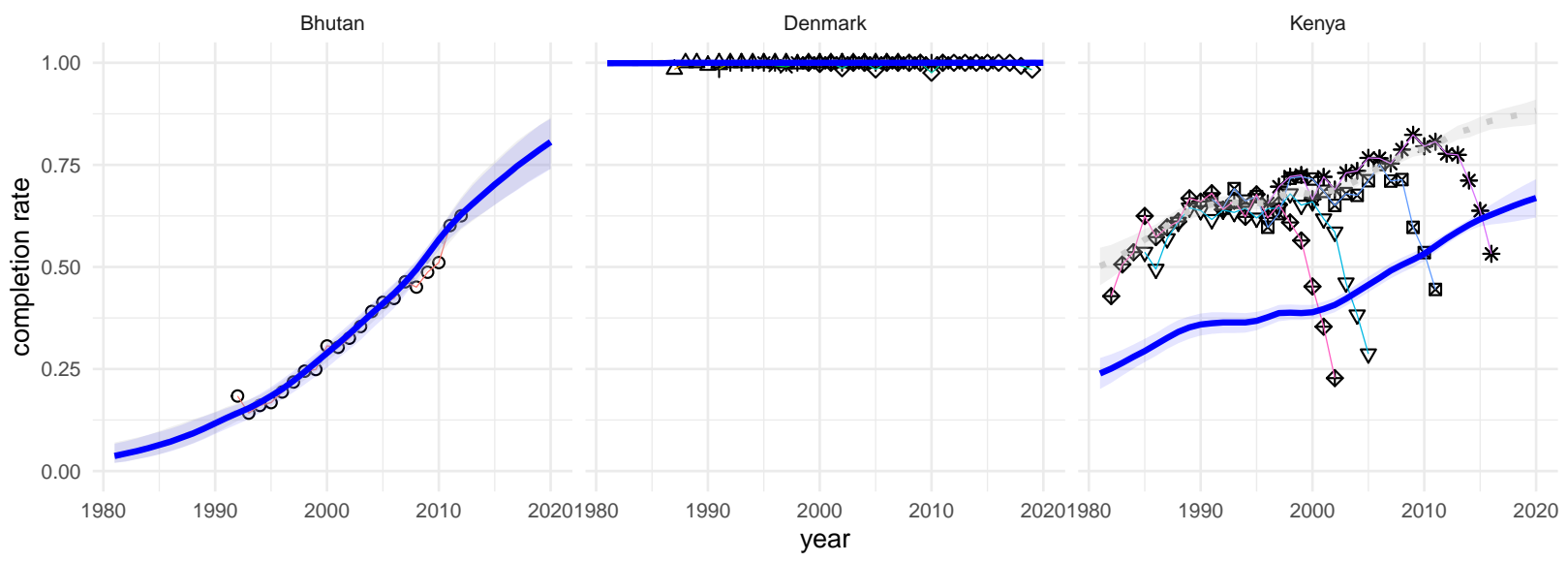

(b) Lower secondary

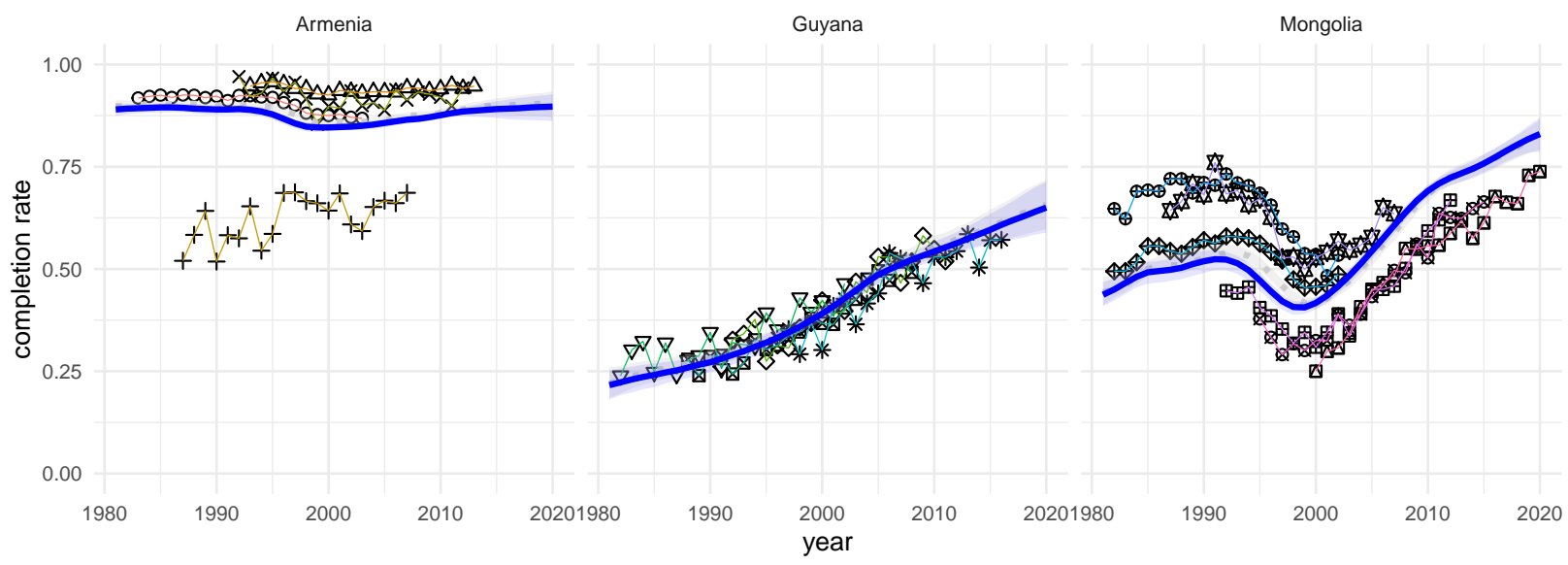

(c) Upper secondary

Figure 6: Country projection by level. The solid and dashed lines denote the Completion Rate indicator and ultimate completion respectively. 
Table 2: ABC random test set validation results.

\begin{tabular}{|c|c|c|c|c|c|c|c|c|c|}
\hline Level & Sex & $\begin{array}{r}\text { MSE } \times \\
100 \\
(\mathrm{ABC})\end{array}$ & $\begin{array}{r}\text { MSE } \times \\
100 \\
(\text { simple })\end{array}$ & $\begin{array}{r}\text { MAE } \times \\
100 \\
(\mathrm{ABC})\end{array}$ & $\begin{array}{r}\text { MAE } \times \\
100 \\
(\text { simple })\end{array}$ & $\begin{array}{r}\text { Bias } \times \\
100\end{array}$ & $\begin{array}{c}\text { Coverage } \\
(\mathbf{8 0 \%})\end{array}$ & $\begin{array}{c}\text { eCoverag } \\
(90 \%)\end{array}$ & $\begin{array}{c}\text { eCoverage } \\
(95 \%)\end{array}$ \\
\hline prim & female & 0.25 & 0.38 & 2.83 & 3.51 & 0.28 & 0.87 & 0.94 & 0.97 \\
\hline prim & male & 0.23 & 0.45 & 2.94 & 3.82 & 0.54 & 0.88 & 0.94 & 0.97 \\
\hline prim & total & 0.21 & 0.40 & 2.56 & 3.38 & 0.39 & 0.88 & 0.95 & 0.97 \\
\hline lsec & female & 0.26 & 0.35 & 3.29 & 3.83 & 0.31 & 0.86 & 0.94 & 0.97 \\
\hline lsec & male & 0.34 & 0.49 & 3.90 & 4.68 & 0.47 & 0.86 & 0.94 & 0.97 \\
\hline lsec & total & 0.25 & 0.35 & 3.20 & 3.88 & 0.27 & 0.88 & 0.94 & 0.98 \\
\hline usec & female & 0.38 & 0.41 & 4.07 & 4.21 & 0.11 & 0.86 & 0.95 & 0.98 \\
\hline usec & male & 0.51 & 0.52 & 4.97 & 5.19 & 0.09 & 0.87 & 0.94 & 0.98 \\
\hline usec & total & 0.39 & 0.42 & 4.00 & 4.20 & 0.15 & 0.87 & 0.95 & 0.98 \\
\hline
\end{tabular}

The out-of-sample validation exercises performed suggests that the model outperforms the simple specification and is fairly well calibrated. Additional supporting in-sample visuals and commentary are provided in Appendix B.4.

\subsection{Posterior parameter estimates}

In addition to the posterior estimates of the outcome, we examine the estimates for specific model parameters relating to phenomena of substantive interest. Figure 7 summarises the distributions of the median estimates for survey bias, late completion, and age-misreporting.

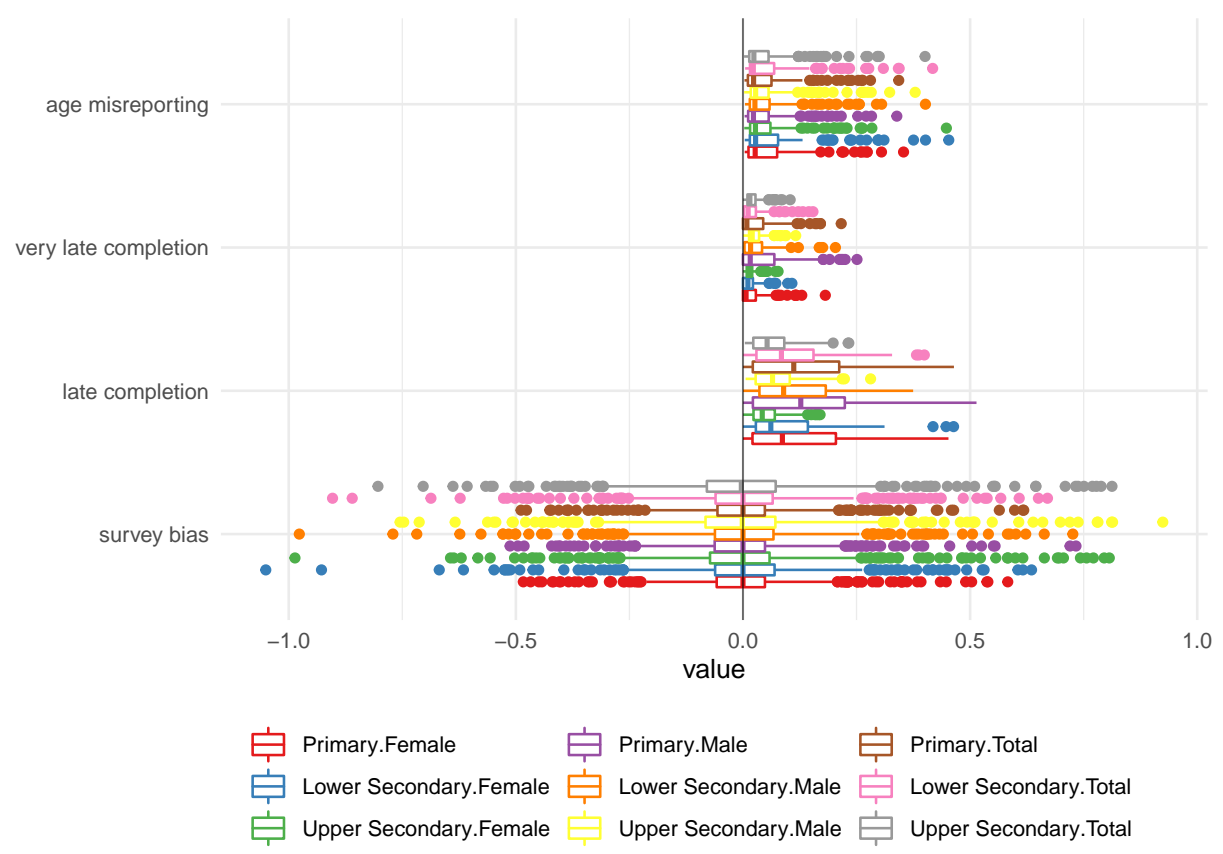

Figure 7: Distribution of posterior medians of survey bias, late completion, and age-misreporting, probit scale.

Recall that the two-stage specification of the age-misreporting effect: whether it occurs, and if, then how strongly. Figure 7 displays the latter, the strength of the age-misreporting effect as it applies to the relevant 
observations. That the effect is of similar magnitude across education levels is not entirely surprising, because the age reporting of those without any schooling (putatively the group most likely to misreport) will distort the denominator of primary, lower, and upper secondary completion equally. In terms of its magnitude, it is clear that this effect cannot be neglected. It can exceed the typical magnitude of bias of individual surveys, and is comparable in magnitude to the distortion arising from late secondary completion.

Turning to the degree of late completion, we can see that there is large variation between countries, but largely around fairly high levels. Values in Figure 7 are shown on the scale of $\kappa$. Because of the floor and ceiling effect at $0 \%$ and $100 \%$ respectively, the translation of the lateness parameter to the original completion rate scale depends on the level of completion it modifies due to the nonlinearity of the probit function.

To illustrate the estimated impact of late completion in the real space, in Figure 8 we plot histograms of the percentage difference in completion between the top and bottom of the age bracket for the combined female and male populations across all country-years. That is, we plot $\Phi\left(\kappa_{c, y}\right)-\Phi\left(\kappa_{c, y}+\phi_{a_{3}, c}\right)$. In many countries, the late completion effect is small, but delays of $10 \%$ or more are prevalent in the primary and lower secondary estimates. Extreme delays of $20 \%$ or more are found in 24 countries, suggesting that major delays in schooling are deeply embedded in those education systems.

The finding that late completion is lower at higher levels of schooling is interesting. A priori, it might be expected that a longer school career up to that point would have created more opportunities for delays. Also, the next higher level, tertiary education, is completed by many much later than the 'theoretical' age for timely completion. Countering these effects, the fact that in our results there is less late completion at the upper secondary level suggests that late completers at lower levels drop out - or are pushed out.
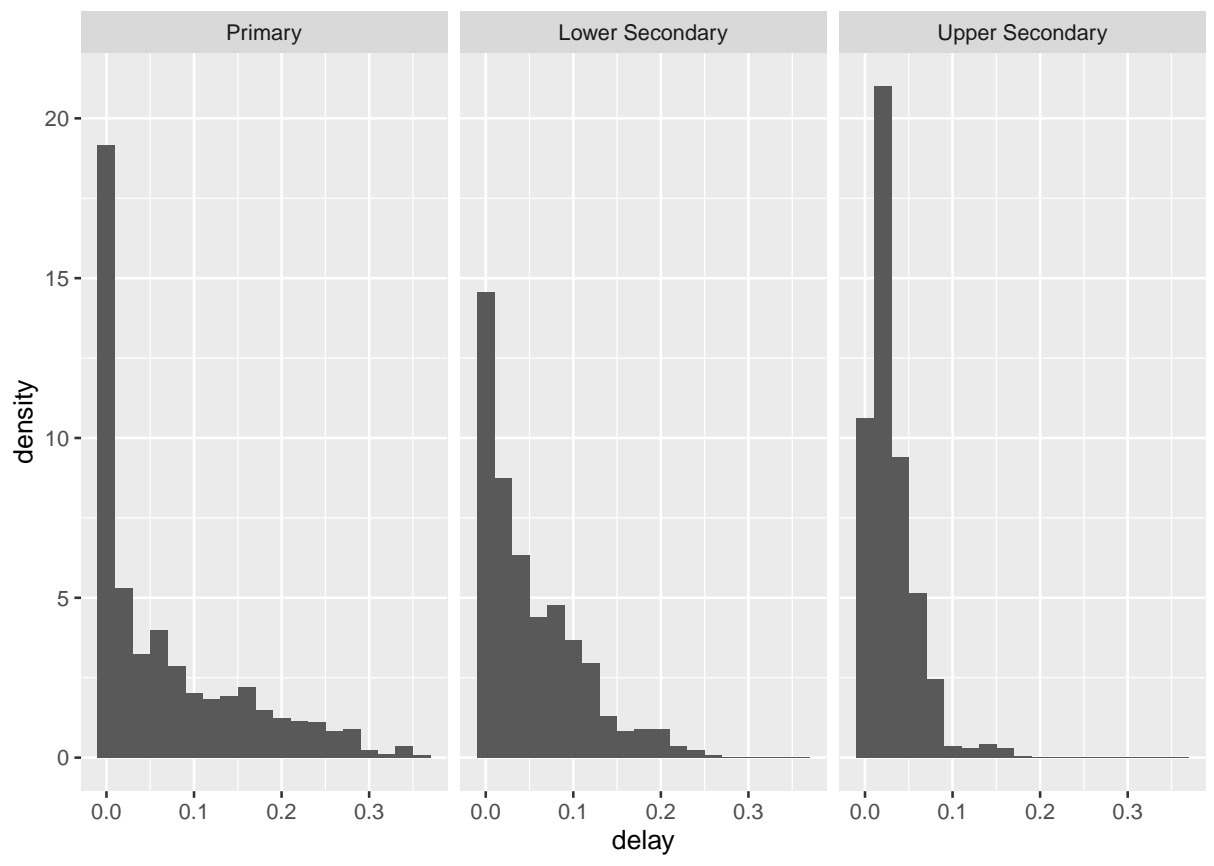

Figure 8: Distribution of late completion delays with the indicator age bracket on the real scale.

Because the model can only estimate relative survey bias, the estimates for the survey bias terms centre on zero. The presence of heavily biased surveys was expected and encoded through the Cauchy distribution but the high frequency of extreme bias illustrated by Figure 7 is still surprising. This calls for great caution in interpreting any survey-based education indicators in countries where only a single survey has been conducted.

Figure 9 displays the uncertainty around individual bias estimates, by survey series. Note that this plot includes only the survey bias estimates from the combined male and female population models and excludes country-specific non-standard surveys. We see that the conclusion that some individual surveys suffer heavy 
bias is confirmed with great confidence. However, it is also apparent that apart from MICS6 which displays fairly consistent positive bias, there is no discernible systematic difference between DHS and MICS families, or specific waves: the estimated systematic bias common to surveys of a given type is practically indistinguishable from zero. We also notice that censuses do not seem to gravitate towards lower bias values or provide any indication of being a 'gold standard'.

It is possible that the 'systematic bias' of household survey estimates of school completion is largely shared across survey designs. In other words, to the extent that some low-education groups are missing from sampling frames, such as nomads or children in orphanages, they tend to be missed by household surveys in general. If this is the case, the implication is that 'better' household surveys may not be sufficient to capture invisible groups, and that altogether, alternative approaches to complement them may be needed.

\section{Conclusion}

In this work, we present a novel method to estimate school completion rates for the official SDG 4 'Completion Rate' indicator. This method, the Adjusted Bayesian Completion Rate (ABC) model, consolidates reconstructed survey data and addresses the significant data challenges of age-misreporting and late completion in order to estimate the underlying true completion rate. Such steps are necessary given that the ABC model represents the first attempt at modelling school completion in the youth population as opposed to waiting a number of decades and modelling final adult completion. Comparing the model with a simpler base case validates the presented specification.

We recognize, however, that global model-based estimates of development indicators should not be mistaken for 'the real thing'. This is especially true when they are used to assess the attainment of specific time-bound targets. In the present case, at the time of writing, the most recent surveys (for a handful of countries) collected data in 2018 and 2019. The median most recent survey year across countries is 2015. We do believe our estimates for 2019, say, to be the 'best guess' for the situation given past dynamics.

In the immediate future, however, there is much uncertainty regarding the effects the COVID-19 pandemic will have on education. We believe that our model's current short-term projections can act as a useful baseline with which to answer the 'what could have been' question regarding the impact of COVID-19. Further, as new surveys are conducted in the coming years, we expect our model to continue to provide good estimates given that it has proven to be flexible in responding to any shocks that may manifest as evidenced by examples in Figure 6 . That said, this model specification would not explicitly capture a trend break in school completion due to COVID-19 given that the drift parameter is shared by all years.

Similarly, even though there have yet to be any signs that the adoption of the SDG agenda did actually induce a major trend break, if one were to occur, it would not manifest as an explicit change in long-term drift and instead would be found as a realignment though a shock in the residuals.

Two key challenges are identified. At a fundamental level, absent unbiased sources of estimates for at least some countries, we can only estimate the relative bias of different surveys. The fact that all available surveys may be undersampling educationally-distinctive population groups cannot at present be accounted for without incorporating strong a priori assumptions about this general bias. In addition, for the 15 countries with only a single survey, the relative bias specification cannot attribute any survey bias uncertainty given the lack of consistent survey wave or type level patterns in the bias structure.

Secondly, while the principal rationale for the $C R$ indicator was that individuals in the reference age brackets could be assumed to have completed the school level in question, our results show that severe amounts of very late completion, even five years or more above the theoretical graduation age, are common. At the same time, however, both the amount and age pattern of late completion differs greatly across countries and levels of schooling. This represents a key challenge to the model specification, where all these patterns must be captured parsimoniously. There is a trade-off between fitting late completion and the ability to identify recent decline in ultimate completion. The current specification may err on the side of identifying 

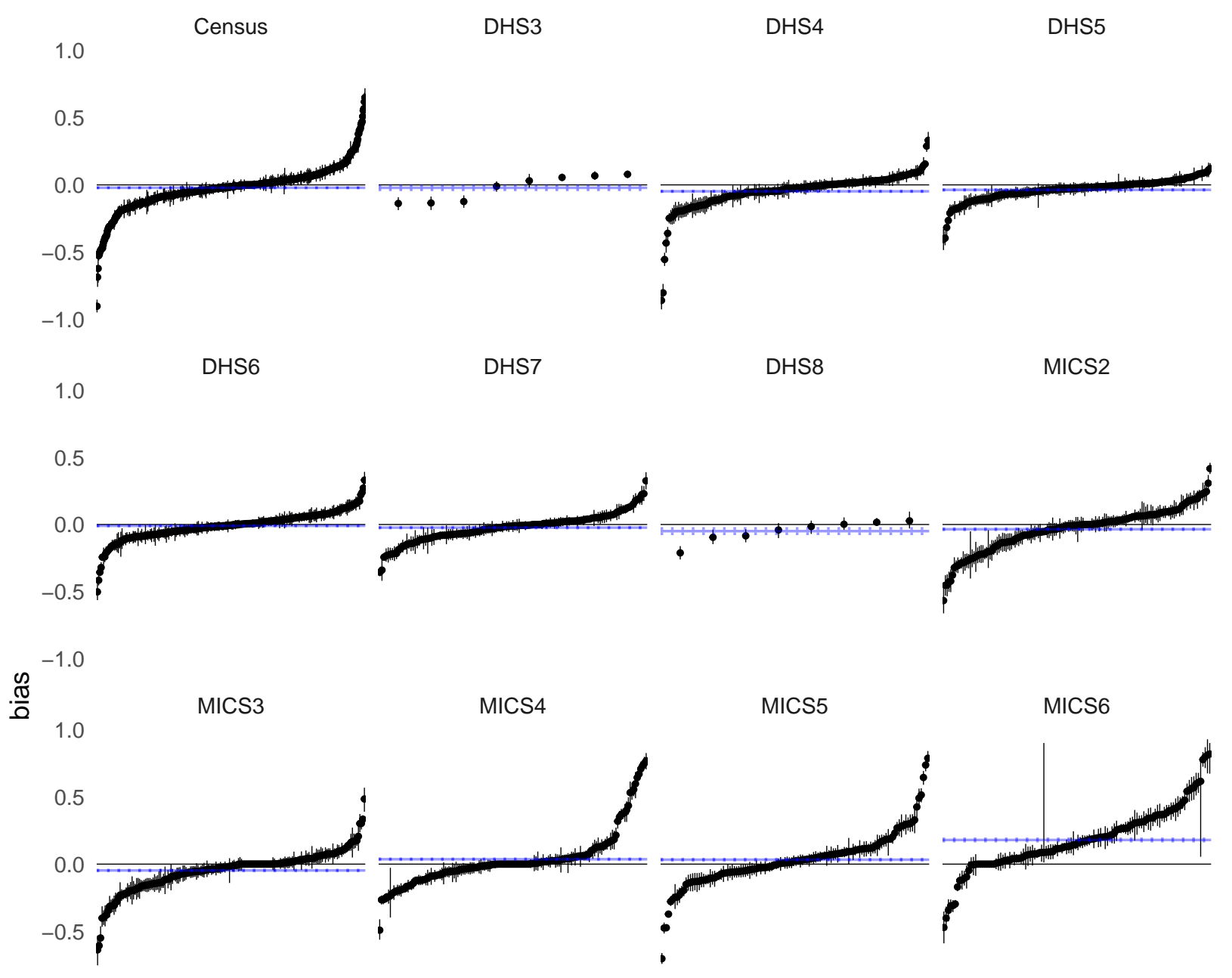

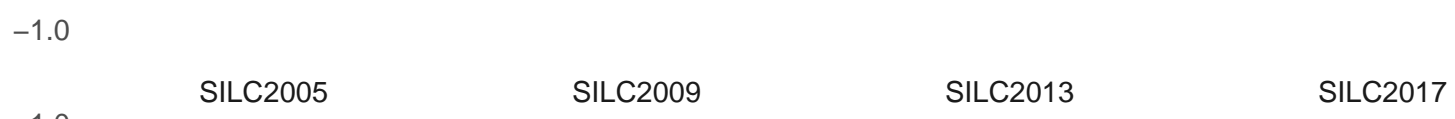

1.0

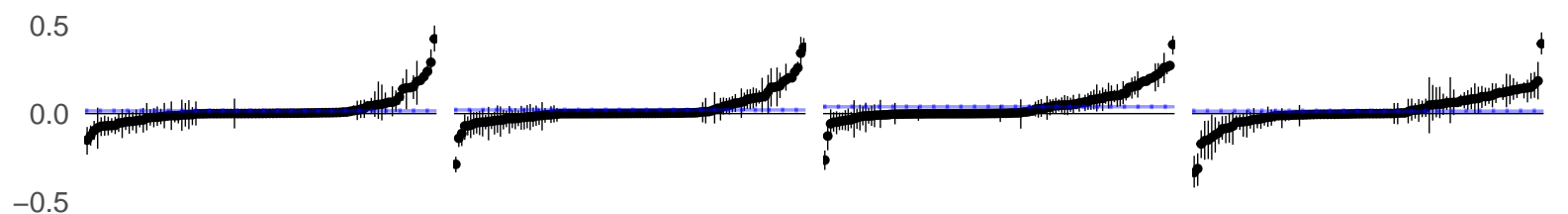

$-1.0$

Figure 9: Posterior distribution of individual survey bias terms, probit scale. Blue lines: median (solid), 10th and 90th percentile (dotted). 
declines, as several cases can be identified where, given contextual background knowledge, projected declines are recognised as spurious consequences of atypical late completion patterns.

At some level, the finding of such widespread and considerable late completion is disappointing, because the rationale behind defining the completion rate indicator with respect to an age group several years above the nominal graduation age was precisely to minimise this effect. The true ultimate completion rate in a given cohort can be observed at some significantly higher age such as 30 years such that further school completion can safely be assumed to be statistically negligible. The age bracket 3 to 5 years above the nominal age for the final grade was assumed to be a reasonably good approximation. Our results clearly show that the completion rate indicator thus defined can not be interpreted as a proxy for ultimate cohort completion. Instead, it should be recognised as measuring what might be termed 'reasonably timely completion'.

\section{Acknowledgements}

We would like to thank the members of the Technical Cooperation Group on the Indicators for SDG 4 for their input. We also thank Monica Alexander for her comments and feedback throughout this project. This paper is based on data from the Demographic and Health Surveys (DHS), Multiple Indicator Cluster Surveys (MICS), Eurostat, EU Statistics on Income and Living Conditions (EU-SILC), Integrated Public Use Microdata Series (IPUMS), Encuesta Permanente de Hogares (EPH), Pesquisa Nacional por Amostra de Domicílios (PNAD), Encuesta de Caracterización Socioeconómica Nacional (CASEN) and the China Family Panel Studies (CFPS) from the years 1999-2019. The responsibility for all conclusions drawn from the data lies entirely with the authors.

\section{References}

Alexander, M. (2020) Distortr: Temporal Smoothing Methods for Demographic Time Series. Available at: https://github.com/MJAlexander/distortr.

Alexander, M. and Alkema, L. (2018) Global estimation of neonatal mortality using a bayesian hierarchical splines regression model. Demographic Research, 38, 335-372. DOI: 10.4054/DemRes.2018.38.15.

Alkema, L. and New, J. R. (2014) Global estimation of child mortality using a bayesian b-spline biasreduction model. The Annals of Applied Statistics, 8, 2122-2149. Institute of Mathematical Statistics. DOI: 10.1214/14-aoas768.

Alkema, L., Chou, D., Hogan, D., et al. (2016) Global, regional, and national levels and trends in maternal mortality between 1990 and 2015, with scenario-based projections to 2030: A systematic analysis by the un maternal mortality estimation inter-agency group. The Lancet, 387, 462-474. Elsevier. DOI: 10.1016/S01406736(15)00838-7.

Bengtsson, H. (2020a) A unifying framework for parallel and distributed processing in $\mathrm{r}$ using futures. Available at: https://arxiv.org/abs/2008.00553.

Bengtsson, H. (2020b) Future: Unified Parallel and Distributed Processing in R for Everyone. Available at: https://github.com/HenrikBengtsson/future.

Carpenter, B., Gelman, A., Hoffman, M. D., et al. (2017) Stan: A probabilistic programming language. Journal of Statistical Software, Articles, 76, 1-32. DOI: 10.18637/jss.v076.i01.

Carvalho, C. M., Polson, N. G. and Scott, J. G. (2009) Handling Sparsity via the Horseshoe. eds D. van Dyk and M. Welling. Proceedings of machine learning research. Hilton Clearwater Beach Resort, Clearwater Beach, Florida USA: PMLR. Available at: http://proceedings.mlr.press/v5/carvalho09a.html.

Chung, Y., Rabe-Hesketh, S., Dorie, V., et al. (2013) A nondegenerate penalized likelihood estimator for 
variance parameters in multilevel models. Psychometrika, 78, 685-709. Springer. DOI: 10.1007/s11336-013$9328-2$.

Eurostat (2005-2017) EU statistics on income and living conditions (various) [datasets]. Eurostat. Available at: https://ec.europa.eu/eurostat/web/main/data/database.

Gabry, J., Simpson, D., Vehtari, A., et al. (2019) Visualization in bayesian workflow. Journal of the Royal Statistical Society: Series A (Statistics in Society), 182, 389-402. Wiley. DOI: 10.1111/rssa.12378.

Gelman, A. and Rubin, D. B. (1992) Inference from iterative simulation using multiple sequences. Statistical Science, 7, 457-472. Institute of Mathematical Statistics. Available at: http://www.jstor.org/stable/2246093.

IBGE (2007-2015) Pesquisa nacional por amostra de domicílios [datasets]. Instituto Brasileiro de Geografia e Estatística. Available at: http://observatorio.ministeriodesarrollosocial.gob.cl/index.php.

ICF (2000-2018) Demographic and health surveys (various) [datasets]. Rockville, MD: ICF; Funded by USAID. Available at: https://dhsprogram.com/.

INDEC (2004-2012) Encuesta permanente de hogares [datasets]. Instituto Nacional de Estadística y Censos de la República Argentina. Available at: https://www.indec.gob.ar/indec/web/Institucional-IndecBasesDeDatos.

Landau, W. M. (2018) The drake r package: A pipeline toolkit for reproducibility and high-performance computing. Journal of Open Source Software, 3. DOI: 10.21105/joss.00550.

Landau, W. M. (2020) Drake: A Pipeline Toolkit for Reproducible Computation at Scale. Available at: https://CRAN.R-project.org/package=drake.

Ministerio de Desarrollo Social y Familia (2000-2015) Encuesta de caracterización socioeconómica nacional [datasets]. Ministerio de Desarrollo Social y Familia. Available at: http://observatorio. ministeriodesarrollosocial.gob.cl/index.php.

Minnesota Population Center (2020) Integrated public use microdata series, international: Version 7.3 [datasets]. Minneapolis, MN: IPUMS. DOI: 10.18128/D020.V7.2.

Peking University Open Research Data (2010-2014) China family panel studies [datasets]. Peking University Open Research Data. Available at: https://opendata.pku.edu.cn/dataverse/CFPS?language=en.

Piironen, J. and Vehtari, A. (2017) Sparsity information and regularization in the horseshoe and other shrinkage priors. Electronic Journal of Statistics, 11. Institute of Mathematical Statistics. DOI: 10.1214/17ejs1337si.

Schubert, M. (2019) clustermq enables efficient parallelization of genomic analyses. Bioinformatics, 35, 4493-4495. DOI: 10.1093/bioinformatics/btz284.

Schubert, M. (2020) Clustermq: Evaluate Function Calls on Hpc Schedulers (Lsf, Sge, Slurm, Pbs/Torque). Available at: https://mschubert.github.io/clustermq/.

Stan Development Team (2020a) Brief Guide to Stan's Warnings. Available at: https://mc-stan.org/misc/ warnings.html.

Stan Development Team (2020b) Prior Choice Recommendations. Available at: https://github.com/stan$\mathrm{dev} /$ stan/wiki/Prior-Choice-Recommendations.

Stan Development Team (2020c) Rstan: R Interface to Stan. Available at: https://CRAN.R-project.org/ package $=$ rstan .

The Demographic and Health Surveys Program (2012) Demographic and Health Survey Sampling and Household Listing Manual. ICF International. Available at: https://dhsprogram.com/pubs/pdf/DHSM4/ DHS6_Sampling_Manual_Sept2012_DHSM4.pdf.

UNESCO (2016) Global education monitoring report 2016 box 14.2. UNESCO. Available at: https://unesdoc. 
unesco.org/ark:/48223/pf0000245752.

UNESCO Institute of Statistics (2020) Indicator 4.1.2: Completion rate (primary education, lower secondary education, upper secondary education). UNESCO Institute of Statistics. Available at: https://unstats.un.org/ sdgs/metadata/files/Metadata-04-01-02.pdf.

UNICEF (1999-2019) Multiple indicator cluster survey (various) [datasets]. UNICEF. Available at: https: //mics.unicef.org/.

Vehtari, A., Gelman, A. and Gabry, J. (2017) Practical bayesian model evaluation using leave- one-out cross-validation and waic. Statistics and Computing, 27, 1413-1432. DOI: 10.1007/s11222-016-9696-4.

Vehtari, A., Gabry, J., Magnusson, M., et al. (2020) Loo: Efficient Leave-One-Out Cross-Validation and Waic for Bayesian Models. Available at: https://CRAN.R-project.org/package=loo.

Vehtari, A., Gelman, A., Simpson, D., et al. (2020) Rank-normalization, folding, and localization: An improved $\widehat{R}$ for assessing convergence of mcmc. Bayesian Analysis. Institute of Mathematical Statistics. DOI: 10.1214/20-ba1221.

Xie, Y. and Lu, P. (2015) The sampling design of the china family panel studies (cfps). Chinese Journal of Sociology, 1, 471-484. DOI: 10.1177/2057150X15614535.

Yao, Y., Vehtari, A., Simpson, D., et al. (2017) Using stacking to average bayesian predictive distributions. Bayesian Analysis. DOI: 10.1214/17-BA1091.

\section{A Model Details}

Putting all errors and adjustments together, we model the empirical observations $K_{i}$ of probit completion relating to age $a[i]$, country $c[i]$, year $y[i]$ and originating from survey $s[i]$ as resulting from: the 'true' probit completion $\kappa_{c[i], y[i]}$, survey bias $\beta_{s[i]}$, a distortion due to age-misreporting $\tau_{c[i]}$ and the late (relative to $a_{5}$ ) completion term $\phi_{a[i], c[i]}$, and a total error variance consisting of sampling and non-sampling error with variances $\nu_{i}^{2}$ and $\omega_{a[i], s[i]}^{2}$ respectively:

$$
K_{i} \mid \kappa_{c[i], y[i]}, \beta_{s[i]}, \tau_{c[i]}, \phi_{a[i], c[i]}, \nu_{i}, \omega_{a[i], s[i]} \sim \mathcal{N}\left(\kappa_{c[i], y[i]}+\beta_{s[i]}-\tau_{c[i]} \cdot \mathbb{1}_{5 \mid a[i]}+\phi_{a[i], c[i]}, \quad \nu_{i}^{2}+\omega_{a[i], s[i]}^{2}\right),
$$

The model can be divided into two stages. First is the process model for the underlying 'true' completion rates, $\kappa_{c[i], y[i]}$. The second details how the underlying completion rates relate to the observed data. This is divided into adjustments for late completion and those for various data considerations.

Our estimation is conducted within a Bayesian framework. For the most part, we assign vaguely-informative priors. The following is a discussion of the rationale behind our choices.

\section{A.1 Process Model}

We assume the underlying 'true' values follow an $\operatorname{ARIMA}(1,1,0)$ process with drift:

$$
\begin{aligned}
\Delta \kappa_{c, y}=\kappa_{c, y}-\kappa_{c, y-1} & =\gamma_{c}+\rho_{c} \Delta \kappa_{c, y-1}+\epsilon_{c, y}, \\
\epsilon_{c, y} \mid \sigma_{\epsilon} & \sim \mathcal{N}\left(0, \sigma_{\epsilon}^{2}\right)
\end{aligned}
$$

with the following priors: 


$$
\begin{aligned}
\sigma_{\epsilon} & \sim \operatorname{Gamma}(2,0.1), \\
\rho_{c} \mid \tau, \lambda_{c} & \sim \mathcal{N}^{+}\left(0, \tau^{2} \lambda_{c}^{2}\right), \\
\tau \mid \tau_{0} & \sim \mathcal{N}^{+}\left(0, \tau_{0}^{2}\right), \\
\lambda_{c} & \sim t_{4}^{+}(0,1) \\
\gamma_{c} \mid \alpha_{\gamma}, \beta_{\gamma} & \sim \log -\operatorname{normal}\left(\alpha_{\gamma}, \beta_{\gamma}\right), \\
\alpha_{\gamma} & \sim \mathcal{N}(0,1), \\
\beta_{\gamma} & \sim \mathcal{N}^{+}(0,1) .
\end{aligned}
$$

With respect to the country-specific drift $\gamma_{c}$, we specify a log-normal distribution with a hyperprior setup. The choice of a distribution constrained to be non-negative reflects the expectation of a positive trend in the long run, in line with the objectives of SDG 4. Further, for a country currently far from zero, a negative long-term drift implying eventual convergence to zero completion is not logically plausible.

Any short- to medium-term negative trends are then modeled with $\epsilon_{c, y}$ and the autoregressive coefficient $\rho_{c}$. Given that significant departures from the long-term trend are not expected in all countries, we use a horseshoe prior on $\rho_{c}$ to shrink the $\rho_{c}$ terms corresponding to the countries without these irregular patterns to zero. The horseshoe prior setup here uses a t-distribution for $\lambda_{c}$ instead of the standard Cauchy distribution to simplify for computation purposes. The lighter tails of the country-specific shrinkage distribution is compensated by a higher variance of the distribution of $\tau$, the global shrinkage distribution. The hyperparameter for the prior variance of $\tau, \tau_{0}$, is selected following the discussion in Piironen and Vehtari (2017). There, the shrinkage factor is defined as $\kappa_{j}=\left(1+n \sigma^{-2} \tau^{2} \lambda_{j}^{2}\right)^{-1}$ and the effective number of nonzero coefficients is $m_{\text {eff }}=\sum_{j=1}^{D}\left(1-\kappa_{j}\right)$ where $n$ is the sample size, $\sigma$ is the error and $D$ is the dimension. If $\lambda_{j} \sim$ Cauchy $^{+}(0,1)$, setting the expected number of nonzero coefficients to a prior guess $p_{0}$ yields the value $\tau_{0}=\frac{p_{0}}{D-p_{0}} \frac{\sigma}{\sqrt{n}}$ for the $\tau$ hyperparameter. Given that we use $\lambda_{c} \sim t_{4}^{+}(0,1)$ instead of $\lambda_{c} \sim$ Cauchy $^{+}(0,1)$ we simulated values to $\kappa$ to select the value $\tau_{0}=0.01$ that corresponds to approximately $30 \%$ as the prior guess for the number of nonzero coefficients.

We allow the scaling of year-over-year residuals to be determined by the data though the prior on said scaling $\sigma_{\epsilon}$ is specifically boundary avoiding to prevent a collapsing scenario. For perspective on what the effects of a given magnitude in the transformed space imply on the outcome scale of percent completion, note that for $C=0.5$, i.e. $50 \%$ completion in year $y$, a 1 percentage point change corresponds approximately to a change in $\kappa$ of \pm 0.025 . Practically speaking, while the $\operatorname{Gamma}(2,0.1)$ prior is well overdispersed, the resulting scaling terms are closer in scale to 0.02 , an entirely plausible value for the jitter term while still allowing for reasonably large shocks.

However, we do acknowledge that in the extreme tails of the probit curve, small amounts of noise in the real space translate to seismic shocks in the probit space. For example, values of 4 and 5 in the probit space correspond to observations of $99.997 \%$ completion and $99.99997 \%$ completion respectively. Ultimately, these values are both indicating universal completion in the real space. However, when modelling in the probit, space, what is really just noise appears to be dramatic shifts in completion. To avoid attempting to model the extreme noise, we impose a cap on extreme values such that if a country observes its maximum transformed value above 2.5 or minimum transformed value below -2.5 (99.4\% and $0.06 \%$ completion respectively), all of its observations are uniformly shifted inwards such that the maximum is now 2.5 or minimum is -2.5 respectively. This reduces the risk of noise having undue influence on model parameters. In post-processing, the extracted true values are shifted back to restore the original levels.

The initial value of $\kappa$ is assigned an uninformative prior, $\kappa_{c, 1980} \sim N(0,10)$ to allow the data to determine the intercept. 


\section{A.2 Late completion}

The age profile is captured in $\phi_{a, c}$ :

$$
\begin{aligned}
\phi_{a, c} & = \begin{cases}\left(a-a_{5}\right) \cdot \lambda_{1 c} \cdot \mathbb{1}_{l} & \text { if } a \in\left\{a_{3}, a_{4}\right\} \\
\min \left(3, a-a_{5}\right) \cdot \lambda_{2 c} \cdot \mathbb{1}_{v l} & \text { if } a \geq a_{5}\end{cases} \\
\lambda_{1 c} \mid \sigma_{\lambda_{1}} & \sim \mathcal{N}^{+}\left(0, \sigma_{\lambda_{1}}^{2}\right) \\
\lambda_{2 c} \mid \sigma_{\lambda_{2}} & \sim \mathcal{N}^{+}\left(0, \sigma_{\lambda_{2}}^{2}\right) \\
\sigma_{\lambda_{1}} & \sim \mathcal{N}^{+}(0,1) \\
\sigma_{\lambda_{2}} & \sim \mathcal{N}^{+}(0,1)
\end{aligned}
$$

A visual equivalent to the $\phi_{a, c}$ specification is provided in Figure 4 . The indicators in $\phi_{a, c}$ reflect the reality that late completion is only estimated if there is an indication of its presence being more than simply noise. Domain knowledge suggests that in highly-developed education systems with close-to-universal completion, significant amounts of completion several years above the standard age are extremely unlikely and should be interpreted as data problems. That is, the grace period offered by the completion rate indicator is considered to successfully capture all reasonably short delays in completion. Here we define close-to-universal completion as a median observed completion rate above 0.95 . In the case of the long-delayed completion parameter, $\lambda_{2 c}$, it is only estimated for those countries with median observed values below 0.95 . In the case of the medium-delay completion parameter, $\lambda_{1 c}$, it is estimated for those countries with median observed values below 0.95 or $\left[a_{3}, a_{5}\right]$ values consistently below $\left[a_{5}, a_{7}\right]$. In other words, we allow for structural medium-delayed completion to be a possibility even in countries with close-to-universal completion if the data suggests that is the case. Late completion $\phi_{a, c}$ is a 'real' effect in the sense that the true completion at ages other than $a_{5}$ really is different and this is not a measurement artefact.

\section{A.3 Data Considerations}

\section{A.3.1 Survey Bias}

There are a number of inherent challenges regarding survey bias using household surveys. If all surveys overestimate school completion, for example because they exclude street children, this shared bias cannot be identified without additional assumptions and/or data. Accordingly, if one survey is actually unbiased, and another biased, but we cannot identify which is unbiased, the model estimate will attenuate the latter bias, but will also 'correct' the relative 'bias' of the former. In other applications of similar models, this is partly remedied either by exploiting prior information regarding the absolute bias of specific surveys (gained from an intensive re-count in a subsample, for instance), or by comparison with a 'gold standard' data source that is assumed to suffer a low bias.

In the present case of school completion rates, no equivalent to the - theoretical - 'gold standard' of a complete vital registration (VR) system for health applications or specialised in-depth studies for maternal mortality rates (MMR) exists. Even censuses may miss important subgroups, such as street children. This issue is particularly consequential for school completion rates as differences in school completion between included and excluded groups are potentially extreme. It is entirely plausible for primary completion to be almost universal among population in households, but close to zero among 'missing children'.

The lack of a gold standard precludes the estimation of the absolute bias in survey-based estimates. Nevertheless, modelling the bias of available surveys relative to each other allows for an unbiased estimation of what would be estimated if surveys of all type were available for every year, even when only a subset or only a single survey actually is. In other words, if series A were consistently lower than series B, then for years in which only observations from series A are available, we may still conclude that this is likely to be 
an underestimate, and that the model estimate should be higher. We thus settle on a relative survey bias structure.

Define $\beta_{c}$ to be the vector of $S$ survey biases $\beta_{s}$ related to country $c$ when $S>1$. Note that when $S=1$, there can be no relative survey bias and thus the estimate for the single survey's bias is zero. Next, let $\beta_{c}^{*}$ be a vector of length $S-1$ attributed to country $c$. Then, we construct the survey bias estimates as follows:

$$
\begin{aligned}
\beta_{c} & =A_{S} \cdot \beta_{c}^{*} \\
A_{S} & =\left[\begin{array}{ccccc}
1 & 0 & 0 & \ldots & 0 \\
-\frac{1}{S-1} & 1-\frac{1}{S-1} & 0 & \ldots & 0 \\
-\frac{1}{S-1} & -\frac{1}{S-1} & 1-\frac{2}{S-1} & & 0 \\
\vdots & \vdots & & \ddots & \\
-\frac{1}{S-1} & -\frac{1}{S-1} & \ldots & -\frac{1}{S-1} & 1-\frac{S-2}{S-1} \\
-\frac{1}{S-1} & -\frac{1}{S-1} & -\frac{1}{S-1} & \ldots & -\frac{1}{S-1}
\end{array}\right] \\
\beta_{c}^{*} \sigma_{\text {bias }} & \sim \operatorname{Cauchy}\left(0, \sigma_{\text {bias }}\right) \\
\sigma_{\text {bias }} & \sim \mathcal{N}^{+}\left(0,0.25^{2}\right)
\end{aligned}
$$

The relative structure of the survey bias induces a sum-to-zero behaviour in the survey bias terms for each country. Equivalently, if one were to directly estimate $S$ survey bias terms for a country with $S$ surveys, one term would be redundant. This redundancy produces ridge-like geometry which impedes sampling in the Bayesian framework. Instead, parameterizing in terms of $\beta_{c}^{*}$, a vector of $S-1$ values, and transforming into the $S$ survey bias terms with the $S \times(S-1) A_{S}$ matrix reduces the degrees of freedom by one as demanded by the sum-to-zero constraint.

The $A_{S}$ matrix used here is designed to serve two distinct purposes. First define $a_{i j}$ to be the elements of $A_{S}$. Notice that for all columns $j, \sum_{i=1}^{S} a_{i j}=0$, thus enforcing the sum-to-zero constraint in $\beta_{c}$. Second, notice that for all rows $i, \sum_{j=1}^{S-1}\left|a_{i j}\right|=1$. This property propagates the Cauchy $\left(0, \sigma_{\text {bias }}\right)$ prior on $\beta_{c}^{*}$ to an implied prior on $\beta_{c}$ using the properties of the Cauchy distribution. The Cauchy distribution has been selected to capture the possibility of extreme outlier surveys, a possibility that is observed in the data. An example of such a survey is presented for Armenia in Figure 6. The model recognizes that the highly biased or erroneous input could be offering relevant information but does not dramatically deviate from the rest of the surveys.

\section{A.3.2 Age-Misreporting Distortion}

The error in observed completion rates for ages divisible by 5 due to age-misreporting, $\tau_{c}$, has the following prior:

$$
\begin{aligned}
\tau_{c} \mid \lambda_{\tau} & \sim \operatorname{Exp}\left(\lambda_{\tau}\right) \\
\lambda_{\tau} & \sim \mathcal{N}^{+}\left(0,50^{2}\right)
\end{aligned}
$$

This specification allows for potentially large distortions if observed in the data. We note that the specification for age-misreporting is simple. A more complex model could perhaps link the magnitude of age-misreporting to the pool of less-educated likely to misreport. For example, if the prevalence of age-misreporting were a function of primary completion, the estimates of primary completion could be incorporated into the specification. However, such a step would mean that the estimation of completion rates at each level could no longer be done independently. However, too little is known to do this convincingly and so we settle on the simple specification above.

Similarly, in some cases it seems as if the adjacent 'almost round' ages report increased primary school completion as a result of losing some of their unschooled who incorrectly place themselves in the round age 
group. However, in other cases the offsetting increase is more diffuse. Given the lack of consistent pattern across countries, the offset is not modeled explicitly as affecting specific ages, but is allowed to be implicitly absorbed in the overall country intercept.

\section{A.3.3 Variance}

The model accounts for the total error variances resulting from the combination of sampling and non-sampling errors, such that observations of completion rates with larger total error variance carry less weight.

The sampling variance $\nu_{i}^{2}$ of a given specific observation is estimated by clustered Jackknife prior, as input into the model. Specifically, the sampling variance of any given observed completion rate $C_{a, y, c, s}$ in year $y$ at age $a$ in country $y$ from survey $s$ is estimated as (omitting indices for clarity):

$$
\widehat{\operatorname{Var}}(C)=\frac{1}{n(n-1)} \sum_{i=1}^{n}\left(C_{i}-C\right)^{2}
$$

where

$$
C_{i}=n C-(n-1) C_{(i)}
$$

Here, $C$ is calculated on the full sample, $C_{(i)}$ is calculated on the sample with the $i^{\text {th }}$ cluster excluded, and $n$ is the total number of clusters. Given the presence of $100 \%$ completion observations in the data, we calculate $C$ with a minor jitter sourced from a Beta $(0.5,0.5)$ prior that serves the dual purposes of moving observations slightly off 1 for the probit transformation as well as creating microscopic (importantly non-zero) variance when using the jackknife. For IPUMS data, in light of the fact that standard errors of the census samples are in any case much smaller than of the surveys, for simplicity the same approach was applied, with 1,000 random 'clusters', instead of customising the process to the specific stratification of each sample.

After computing the sampling variance in the observed space, it is transformed to the probit space by using the delta method as:

$$
\nu_{i}^{2}=\frac{\widehat{\operatorname{Var}}(C)}{\left(f\left(\Phi^{-1}(C)\right)\right)^{2}}
$$

where $f$ and $\Phi^{-1}$ are the density and inverse CDF of the standard normal distribution respectively.

The extent to which observations $d o$ differ with respect to sampling variability across countries, but crucially also across ages, time, and surveys within countries, is shown in Figure 10 for a country with a typical (Mali) and a wide (Belize) spread of estimated standard errors. The conclusion is that not all data points call for an equally close fit by the model. Even for Belize, if the fitted trend missed an observation by 4 percentage points, say, this would stretch credulity for some observations, but could perfectly plausibly be attributed to sampling error for others.

Non-sampling variance $\omega_{a, s}^{2}$ is composed of a base variance $\omega_{s}^{2}$ and an inflation factor capturing increased uncertainty due to reconstruction:

$$
\begin{aligned}
\omega_{a, s}^{2} & =\left(1+0.05 \cdot \max \left(0, a-a_{5}\right)\right) \cdot \omega_{s}^{2} \\
\omega_{s} & \sim \operatorname{Gamma}(2,4)
\end{aligned}
$$

The gamma prior is selected for its boundary avoiding properties. Unlike with total variance, a zero value for non-sampling variance could be consistent with the likelihood given that sampling variance is guaranteed 


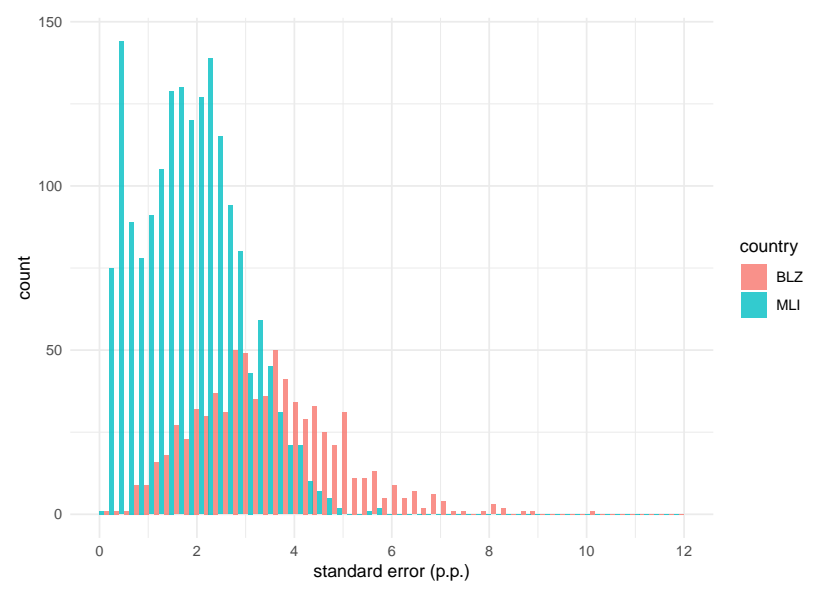

Figure 10: Distribution of estimated sampling standard errors for age-specific completion rates, example countries of Belize and Mali.

to be positive. The gamma distribution reflects the understanding that non-sampling variance is certainly present.

\section{A.4 Population weights}

The customary 'Completion Rate' indicator is the average completion rate over empirically observed individuals in the three-year age interval $\left[a_{3}, a_{5}\right]$, in other words the implicitly population-weighted average. Based on empirically observed completion, it is:

$$
C R_{c, y}=C_{\left[a_{3}, a_{5}\right], c, y}=\sum_{i=3}^{5} \frac{p_{i}}{p_{[3,5]}} C_{a_{i}, c, y} .
$$

Here, $p_{i}$ is the size of the observed population aged $a_{i}, i$ years above the last grade of a given level of schooling, and $p_{[3,5]}$ is the overall population in the age interval $\left[a_{3}, a_{5}\right]$.

Consider a uniform random sample of size 20,000 and a true single year cohort share of $2 \%$, the binomial standard error of the sampled single year cohort share would be $\frac{\sqrt{20,000 \cdot 0.02 \cdot 0.98}}{20,000} \approx 0.001$, or $5 \%$ in relation to the true value of 0.02 . By comparison, only the most extreme cohort-on-cohort growth rates reach $3 \%$, suggesting that random variation in the age distribution will significantly exceed true differences in birth cohort size in all but the largest surveys and extreme fertility settings.

This can be confirmed empirically, as in Figure 11, which for illustration shows the relative size of individual age cohorts in the age interval 10-14 in surveys from the MICS5 series. These profiles are clearly dominated by random fluctuations rather than smooth trends in cohort size driven by population growth. Year on year fluctuations of up to $25 \%$ are unlikely to represent differences in true cohort size. Moreover, differences in the sizes of single year retrospective cohorts will further be distorted by random variation in mortality and migration.

The unweighted average is, therefore, arguably preferable in general, and is certainly more suitable for modelling and projection. This age-standardized 'Completion Rate' indicator $C R_{c, y}^{*}$ is:

$$
C R_{c, y}^{*}=\frac{1}{3} \sum_{a=a_{3}}^{a_{5}} C_{a, c, y}
$$




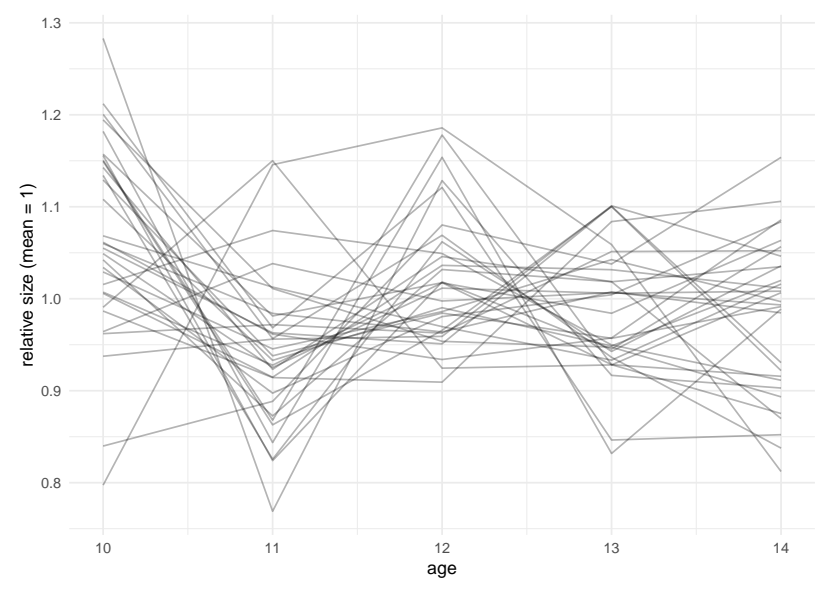

Figure 11: Relative (weighted) size of single-year age groups 10 to 14 in MICS5 surveys.

The equivalent definition for the true completion rates is:

$$
{\widehat{C R^{*}}}_{c, y}=\frac{1}{3} \sum_{a=a_{3}}^{a_{5}} \Gamma_{c, y}=\frac{1}{3} \sum_{a=a_{3}}^{a_{5}} \Phi\left(\kappa_{c, y}+\phi_{a, c}\right)
$$

\section{B Implementation Details}

\section{B.1 Data Sources}

The analysis is based on a consolidated collection of individual-level microdata on school completion. The results presented here are based on 616 distinct surveys from 157 countries.

Specifically, sources include Demographic and Health Surveys (DHS) (ICF, 2000-2018), Multiple Indicator Cluster Surveys (MICS) (UNICEF, 1999-2019), European Union Statistics on Income and Living Conditions (EU-SILC) (Eurostat, 2005-2017), selected other non-standard rhousehold surveys that form the basis for the Global Education Monitoring Report's World Inequality Database on Education (WIDE), and international census samples from the Integrated Public Use Microdata Series (IPUMS) (Minnesota Population Center, 2020). For computational reasons, the IPUMS extracts were limited to 1 million observations each.

Returning to the category of non-standard surveys included in the present analysis, we examined the sample design and microdata of each of the four sets of other surveys leveraged to ensure compatibility with the standard surveys. The four sets of non-standard surveys are:

(a) Argentina: Encuesta Permanente de Hogares (EPH) (INDEC, 2004-2012)

(b) Brazil: Pesquisa Nacional por Amostra de Domicílios (PNAD) (IBGE, 2007-2015)

(c) Chile: Encuesta de Caracterización Socioeconómica Nacional (CASEN) (Ministerio de Desarrollo Social y Familia, 2000-2015)

(d) China: China Family Panel Studies (CFPS) (Xie and Lu, 2015, Peking University Open Research Data (2010-2014))

It is worth noting that all of the countries listed have limited observations sourced through DHS, MICS, and censuses. As such, the addition of non-standard surveys addresses the risk of limited insight on relative sample bias that results from limited survey data. That said, these additional surveys tend to have lower influence in the model due to higher sampling variance resulting from smaller sample sizes and in some cases, less robust sample designs. 


\section{B.2 Computation}

The model was implemented and run in $\mathrm{R}$ version 4.0.2 (2020-06-22) calling on Stan version 2.21.0 on a x86_64-pc-linux-gnu (64-bit) platform. The present exercise draws inspiration from the distortr package (Alexander, 2020) by Monica Alexander that underpins the similarly-motivated models for infant and maternal mortality.

4 chains were run for 3000 iterations each after having discarded 3000 iterations as burn-in, and thinned to 1000 iterations for the computations to reduce the memory footprint. The average runtime for a single chain across all level and sex combinations was 34.09 hours. Chains were run in parallel using the future and clustermq packages (Bengtsson, 2020b, Bengtsson (2020a), Schubert (2020), Schubert (2019)), and the drake package (Landau, 2020, Landau (2018)) was used to ensure reproducibility and assist with version control.

\section{B.3 Convergence}

Convergence was assessed both through Gelman's criterion for potential scale reduction factors (PSRF) and visual inspection of parameter traceplots. We note that values below 1.1 for the PSRF are considered acceptable though the highest PSRF across model runs is 1.04. Figure 12 displays the PSRF values of the 9 parameters with the highest (worst) PSRFs for the estimations for each level and total, female, or male rates. The values are well below the acceptable thresholds. Sample traceplots for the 'worst' parameters corresponding to the model for primary education and both sexes are shown in Figure 13.

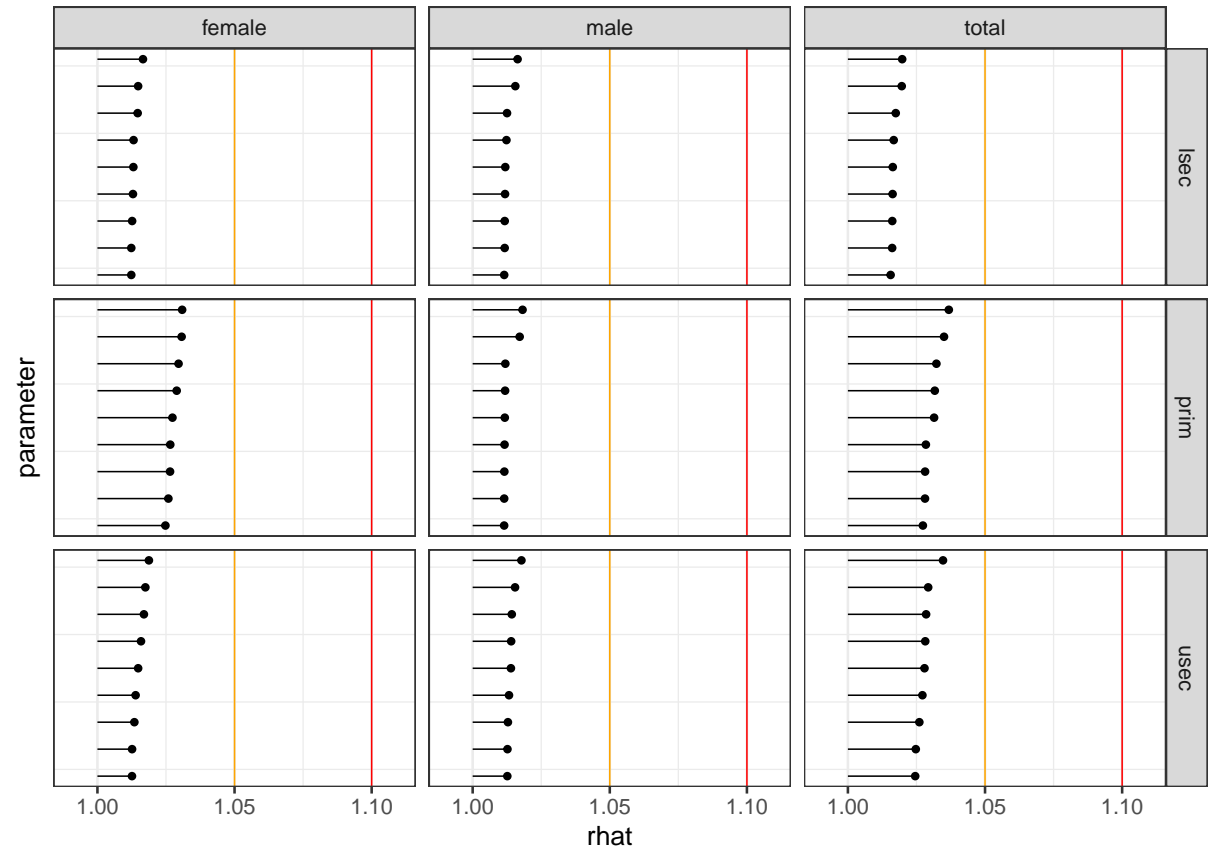

Figure 12: Highest PSRFs by Level and Sex

\section{B.4 Validation}

In addition to the out-of-sample validation exercises described in Section 4.1, we also considered in-sample performance and an approximate leave-one-out cross-validation (LOO-CV) exercise to provide additional insight on the quality of fit. 

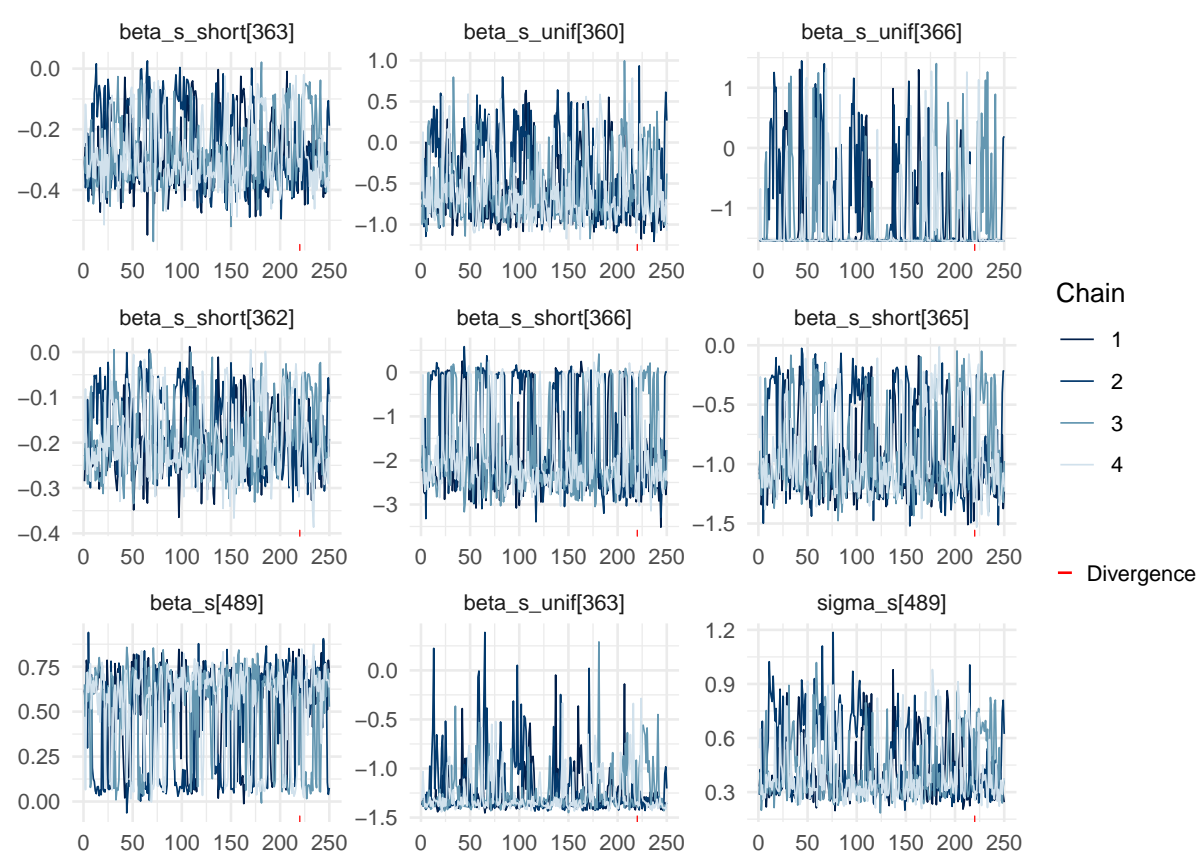

Figure 13: Example Traceplots

Figure 14 shows where a random sample of observations $C_{i}$ distributed evenly across the range of observed values falls within the posterior predictive distribution of $\tilde{C}_{i}$. Note that the observations and distributions presented are in the outcome scale, not the probit scale. This reflects both uncertainty in the central estimate $\hat{C}_{i}$ and simulated draws from the estimated distribution of the residual error. The posterior predictive distributions are consistent with the observations, but without evidence of overfitting. In particular, there is no suggestion that the quality of the fit varies systematically across level, or based on the age at which completers were observed (validating the specification of the age profile). Note that the tail behaviour of the plots, particularly the primary plot, is an extension of the probit transformation used by the model. Specifically, when generating a normal replication in the probit space and subsequently transforming the replications back to the original space, the variability in the extremes compresses as present in the plots.

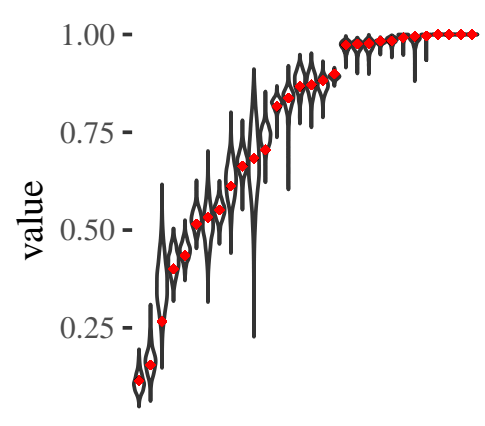

(a) Primary

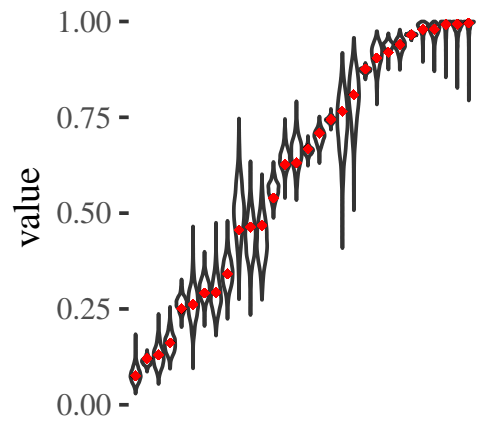

(b) Lower secondary

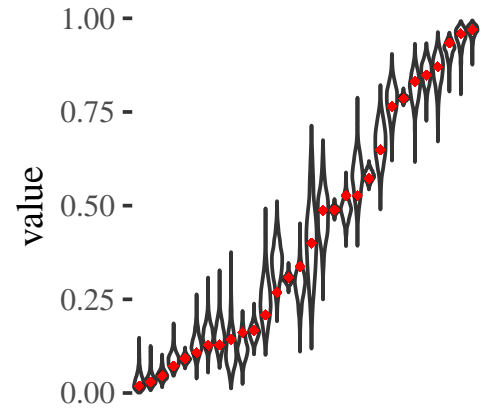

(c) Upper secondary

Figure 14: Posterior predictive distribution for a random sample of observation.

The LOO-CV exercise found that the model seems reasonably well-calibrated (Gabry et al., 2019, Vehtari and Gabry et al. (2020), Vehtari et al. (2017), Yao et al. (2017)). There is some suggestion that the model 
slightly overdisperses but that is not unexpected when considering the diversity in observed values and trends present across levels. The PSIS diagnostics are summarised in Table 3. The percentage of points with $k>0.7$ is fairly small. As has been the trend, the model seems to perform best on the primary school data. The PSIS diagnostic tends to identify points that are outlying or possibly influential. Given that certain model parameters, notably age-reporting and late completion, rely on select few points for estimation, we expect there to be a baseline number of influential points picked up by this test. Concretely, for a country with few surveys, the $a_{3}$ and $a_{4}$ observations entirely determine the late completion parameter and so in a LOO scenario, we expect these points to be flagged. To given a sense of the percentage of outlying points, we remove these points from the list of $k>0.7$ points and recompute the percentage of influential and outlying points.

Table 3: Pareto-k diagnostics.

\begin{tabular}{llrr}
\hline Level & Sex & $\mathbf{k}>\mathbf{0 . 7}$ & $\mathbf{k}>\mathbf{0 . 7}$ (excluding known \\
points)
\end{tabular}

